\title{
Hybrid Reynolds-Averaged / Large Eddy Simulation of a Cavity Flameholder; Assessment of Modeling Sensitivities
}

\author{
R. A. Baurle * \\ NASA Langley Research Center, Hampton, Va 23681
}

\begin{abstract}
Steady-state and scale-resolving simulations have been performed for flow in and around a model scramjet combustor flameholder. The cases simulated corresponded to those used to examine this flowfield experimentally using particle image velocimetry. A variety of turbulence models were used for the steady-state Reynoldsaveraged simulations which included both linear and non-linear eddy viscosity models. The scale-resolving simulations used a hybrid Reynolds-averaged / large eddy simulation strategy that is designed to be a large eddy simulation everywhere except in the inner portion (log layer and below) of the boundary layer. Hence, this formulation can be regarded as a wall-modeled large eddy simulation. This effort was undertaken to formally assess the performance of the hybrid Reynolds-averaged / large eddy simulation modeling approach in a flowfield of interest to the scramjet research community. The numerical errors were quantified for both the steady-state and scale-resolving simulations prior to making any claims of predictive accuracy relative to the measurements. The steady-state Reynolds-averaged results showed a high degree of variability when comparing the predictions obtained from each turbulence model, with the non-linear eddy viscosity model (an explicit algebraic stress model) providing the most accurate prediction of the measured values. The hybrid Reynolds-averaged / large eddy simulation results were carefully scrutinized to ensure that even the coarsest grid had an acceptable level of resolution for large eddy simulation, and that the time-averaged statistics were acceptably accurate. The autocorrelation and its Fourier transform were the primary tools used for this assessment. The statistics extracted from the hybrid simulation strategy proved to be more accurate than the Reynolds-averaged results obtained using the linear eddy viscosity models. However, there was no predictive improvement noted over the results obtained from the explicit Reynolds stress model. Fortunately, the numerical error assessment at most of the axial stations used to compare with measurements clearly indicated that the scale-resolving simulations were improving (i.e. approaching the measured values) as the grid was refined. Hence, unlike a Reynolds-averaged simulation, the hybrid approach provides a mechanism to the end-user for reducing model-form errors.
\end{abstract}

\section{Nomenclature}

$\begin{array}{ll}\text { Symbols } & \\ c & \text { speed of sound } \\ C_{1}, C_{2} & \text { user-specified constants for the Larsson sensor } \\ C_{\mu} & \text { constant for the turbulent viscosity } \\ d & \text { distance to the nearest solid surface } \\ d^{+} & \text {non-dimensional "law of the wall" coordinate } \\ F & \text { hybrid RAS/LES blending function } \\ F_{s} & \text { factor of safety used to assess grid convergence } \\ f & \text { generic functional used to assess grid convergence } \\ k & \text { turbulence kinetic energy } \\ \dot{m} & \text { mass flow rate } \\ P & \text { pressure } \\ p & \text { numerical scheme order of accuracy used to assess grid convergence } \\ P r_{t} & \text { turbulent Prandtl number } \\ R_{i i} & \text { autocorrelation function } \\ r & \text { grid refinement factor used to assess grid convergence }\end{array}$

*Aerospace Engineer, Hypersonic Airbreathing Propulsion Branch, Associate Fellow AIAA. 


\begin{tabular}{|c|c|}
\hline$S c_{t}$ & turbulent Schmidt number \\
\hline$T$ & temperature \\
\hline$u, v, w$ & Cartesian velocity components \\
\hline$x, y, z$ & Cartesian coordinates \\
\hline Vol & grid cell volume \\
\hline$\vec{V}$ & velocity vector \\
\hline$\alpha$ & hybrid RAS/LES blending function constant \\
\hline$\delta$ & boundary layer thickness \\
\hline$\Delta$ & SGS filter width or grid spacing \\
\hline $\bar{\triangle}_{x, y, z}$ & $x, y$, or $z$ direction grid spacings averaged over the computational domain \\
\hline$\epsilon$ & small non-zero floating point value \\
\hline$\eta$ & length scale ratio controlling the hybrid RAS/LES blending function or Kolmogorov length scale \\
\hline$\theta$ & argument associated with the Larsson sensor \\
\hline$\kappa$ & von Karman constant or MUSCL parameter \\
\hline$\lambda$ & turbulence length scale (Taylor microscale) \\
\hline$\mu$ & molecular viscosity \\
\hline$\mu_{\text {sgs }}$ & sub-grid viscosity \\
\hline$\rho$ & density \\
\hline$\tau$ & time difference relative to the initial time for the autocorrelation function \\
\hline$\psi$ & sensor function used to blend non-dissipative and dissipative operators \\
\hline$\omega$ & specific turbulence dissipation rate \\
\hline$\vec{\nabla}$ & gradient operator \\
\hline \multicolumn{2}{|c|}{ Subscripts } \\
\hline$i, j$ & computational coordinate indices \\
\hline$\infty$ & freestream or reference value \\
\hline
\end{tabular}

\section{Introduction}

Reynolds-averaged Computational Fluid Dynamics (CFD) codes are the standard high-fidelity numerical tools utilized in the aerospace industry. These tools have revolutionized the research and development practices, which as early as 15-20 years ago relied almost exclusively on extensive wind tunnel testing. Unfortunately, Reynolds-Averaged Simulations (RAS), which attempt to model all of the scales present in turbulent flows, have proven to be deficient in many challenging areas of interest to the aerospace community. Some examples include:

- high lift devices (massive flow separation)

- combusting flows (particularly lean or rich flames near extinction)

- unsteady flows (rotorcraft, aeroacoustics, etc.)

- shock / boundary layer and shock / jet interactions

In general, the limitations associated with the turbulence closure models are the pacing items preventing the use of RAS in these (and other complex) settings as a true predictive tool. ${ }^{1}$

Large Eddy Simulation (LES) methods have the potential to reduce the modeling uncertainty inherent to RAS approaches, since the intent of LES is to resolve the large scale turbulent structures while modeling only the smallest scales. However, the computational expense of wall-resolved LES at Reynolds numbers relevant to engineering

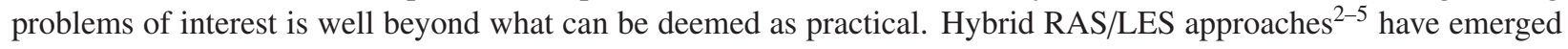
to address this issue, and have provided a rational path forward towards extending LES into practical settings. These methodologies allow LES content to be resolved in areas that require a rigorous modeling approach, while maintaining a more cost effective RAS approach for benign regions of the flow (e.g. attached boundary layers).

The computational expense required for hybrid RAS/LES, while less than that of a full LES, is still formidable when compared with steady-state RAS. Moreover, the numerical algorithms required to resolve the turbulence scales of interest must have low numerical dissipation with minimal dispersive errors (particularly for high-speed flows where shock waves may be present). These observations demand an efficient high-order, low-dissipation numerical framework; a feature not typically required by pure RAS solvers. As a result, the extension of scale-resolving simulation 
approaches (such as hybrid RAS/LES) to engineering problems of interest will only become practical when substantial advancements to both the numerical and physical models have been realized. Towards this end, researchers in the Hypersonic Airbreathing Propulsion Branch (HAPB) and Computational AeroSciences Branch (CASB) of the NASA Langley Research Center have been extending the capabilities of the VULCAN-CFD flow solver ${ }^{6,7}$ for scale-resolving

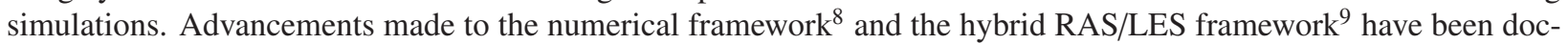
umented for both low and high-speed benchmark flows. The present effort aims to apply this framework to a flow of engineering interest. In particular, this paper describes the results of hybrid RAS/LES performed for a model scramjet combustor flameholder. The flowpath involves a supersonic internal flow passing over a recessed cavity that is internally fueled by an array of ethylene-fueled injection ports. Experimental data available for this configuration include two components of velocity (via particle image velocimetry) ${ }^{10}$ and reacting scalar data (via UV Raman Scattering). ${ }^{11}$ The results described in this paper focus (to a large extent) on the sensitivity of the predictions to numerical modeling choices utilized by the hybrid RAS/LES approach. Comparisons are also made with measured velocities within the cavity flameholder and with steady-state RAS results.

\section{Geometry Description and Flow Conditions}

A schematic of the facility flowpath considered in this study is shown in Fig. 1. An asymmetric facility nozzle provides a continuous flow of Mach 2 (nominal) air to the constant area isolator section of the flowpath. The 7 inch long isolator starts at the $x=0$ inch station and has a 2 inch height (y-direction) and a 6 inch width (z-direction). The $2.5^{\circ}$ divergent portion of the lower wall initiates at the exit of the isolator ( $x=7$ inch station), and the cavity flameholder is located 3 inches downstream of this location. The cavity spans the entire width of the flowpath. The depth of the cavity is 0.65 inches and the length of the cavity floor is 1.815 inches. The aft wall of the cavity has a shallow angle $\left(22.5^{\circ}\right.$ relative to the cavity floor) and houses an array of 11 evenly distributed fuel injection ports. The diameter of each injection port is 0.0078 inches, and the centerline of each port intersects the angled aft cavity surface at the $x, y$ coordinate values of 12.0276 and 0.7703 inches, respectively. The cavity closeout location corresponds to the intersection of the cavity aft wall with the $2.5^{\circ}$ facility lower wall surface.

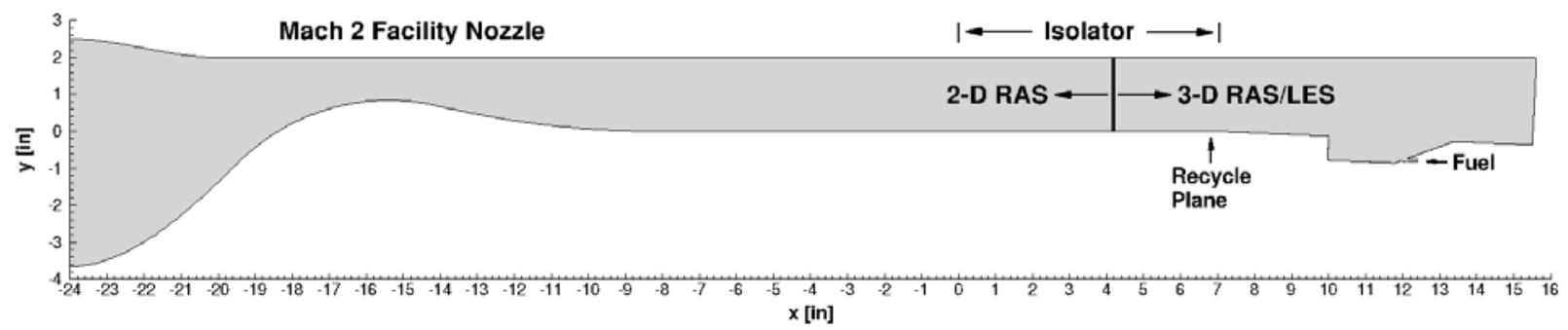

Figure 1: Schematic of the facility flowpath

Table 1: Facility Test Conditions

\begin{tabular}{||c|c|c|c||}
\hline \hline Nominal Conditions & Case 1 & Case 2 & Case 3 \\
\hline \hline Air Mach Number & $2.0^{a}$ & $2.0^{a}$ & $2.0^{a}$ \\
\hline Air $T_{\circ}[\mathrm{K}]$ & 589.0 & 589.0 & 589.0 \\
\hline Air $P \circ[\mathrm{kPa}]$ & 483.0 & 483.0 & 483.0 \\
\hline Fuel Flow Rate [SLPM] & 0.0 & 56.0 & 99.0 \\
\hline Fuel $T_{\circ}[\mathrm{K}]$ & $\mathrm{N} / \mathrm{A}$ & $310.0^{b}$ & $310.0^{b}$ \\
\hline \multicolumn{4}{c}{${ }^{a}$ nozzle design Mach number } \\
${ }^{b}$ estimated bottle temperature
\end{tabular}

The nominal flow conditions considered in the experiments conducted by Tuttle et al. ${ }^{10}$ are given in Table 1. The fuel flow rates, reported in Standard Liters Per Minute (SLPM), are based on a reference state of $273 \mathrm{~K}$ and 1 atmosphere. The facility nozzle geometry was included in the simulated flowpath, so the Mach 2.0 value listed in Table 1 is a nominal value. All of the simulations performed in this work ignored any facility side-wall influences. 
This allowed the exit conditions from an a priori two-dimensional RAS of the facility nozzle (and 4.2 inches of the constant area isolator) to be used as the inflow condition for the three-dimensional simulations performed for the region of interest further downstream. The Mach number and pressure profiles extracted at this interface (see Fig. 1) are shown in Fig. 2. The simulation of the facility nozzle shows a weak shock structure propagating through the isolator as evidenced by the pressure variability along the profile. The boundary layer thickness at this station is 0.25 inches (1/4 of the duct half height) which is nearly $40 \%$ of the cavity depth.

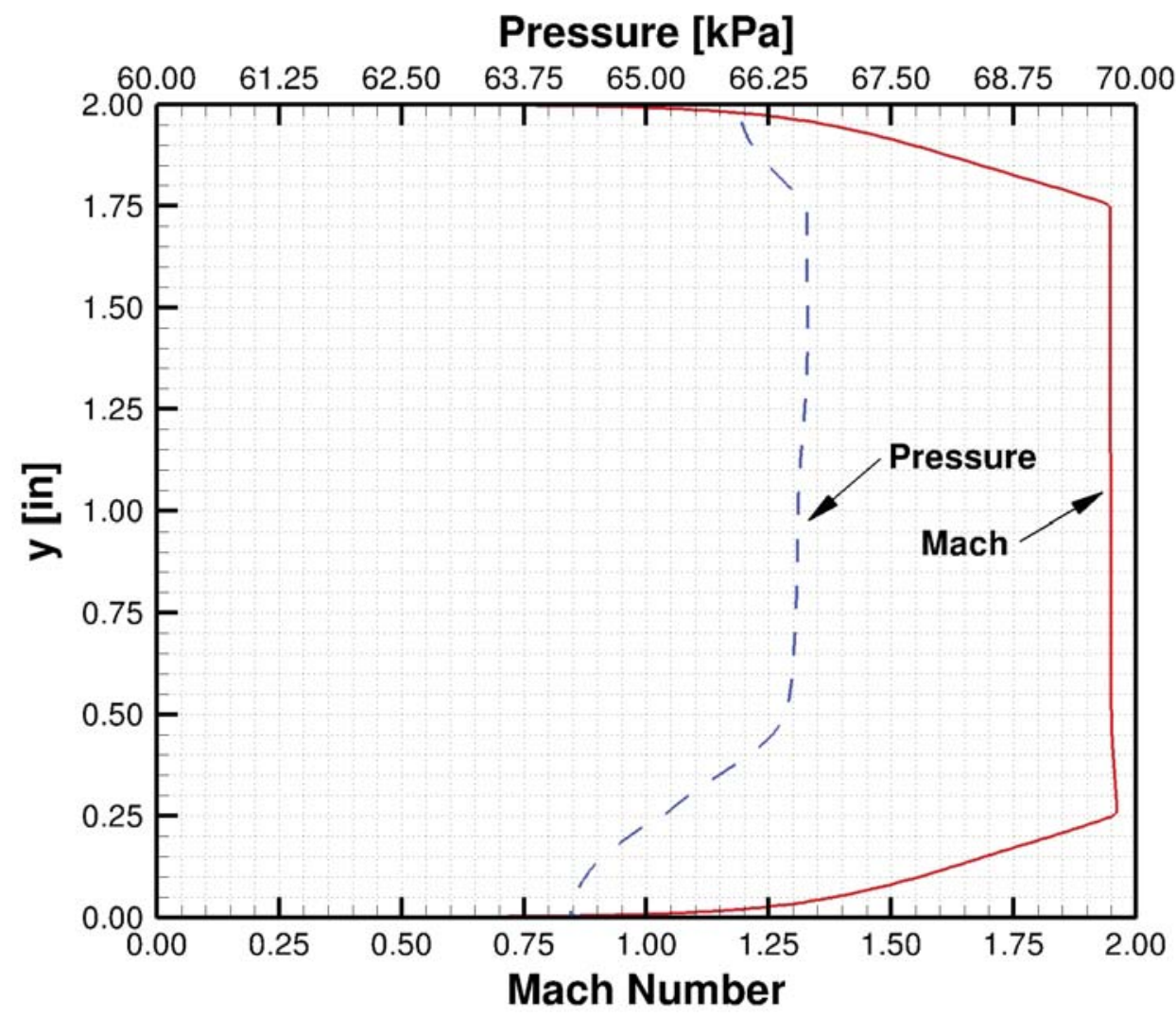

Figure 2: Facility Mach number and pressure profiles at the $x=4.2$ inch station used to prescribe the 3-D RAS and hybrid RAS/LES inflow conditions

\section{Computational Grid Description}

As mentioned previously, the simulations performed for this effort neglected any facility side-wall influences. This allowed the consideration of only a fraction of the facility width, providing for a more efficient means of examining numerical modeling sensitivities while retaining the salient flow features of interest. The smallest spanwise width allowed by the symmetry present in the geometry is a 0.25 inch section that covers the region from the centerplane of an injection port to the gap centerplane between two injector ports. This domain is appropriate for steady-state RAS, with symmetry conditions imposed at each bounding plane. However, symmetry conditions are not appropriate for the unsteady hybrid RAS/LES, since the flow symmetry only applies to the time-averaged flowfield statistics. Hence, these simulations (at a minimum) require a domain that extends from the centerplane of one injector port to the centerplane of an adjacent port (or equivalently one that is bounded by the gap centerplane on either side of an injection port) to allow the specification of periodic conditions at each bounding plane. However, as pointed out previously, the approach boundary layer has a thickness of 0.25 inches which will eventually transition to an even thicker free shear layer over the cavity flameholder. In order to properly accommodate the formation of resolved eddy structures, the largest of which are comparable to the width of the viscous shear layer, a domain larger than 0.5 inches should be considered. More specifically stated, the width should be large enough to ensure that the largest eddy structures become decorrelated when separated by a distance larger than the half-width of the periodic domain. This prompted the consideration of a 1-inch domain width that encompasses two full injection ports. This domain matched that used 
in previous DES research efforts ${ }^{12,13}$ for this configuration.

A sequence of three progressively refined structured grids was created for the simulations. The refinement factor used was a factor of 1.5 in each coordinate direction. The grid attributes closely resembled those utilized for the DES studies performed in Refs. 12 and 13. However, the computational grids generated for this study were fully structured, while the grids in the aforementioned references made use of unstructured hexahedral cells in some regions of the flow. Each grid is comprised of nearly isotropic cells (except in the inner part of the boundary layers where RAS is utilized), and the grid is clustered to all surfaces such that $d^{+}$is approximately unity. The first attribute is driven by the desire to resolve eddy structures in the outer portion of the boundary layers and in free shear regions of the flow. The second is a resolution requirement for accurate RAS modeling of boundary layer flows without resorting to the use of wall functions. The average grid spacing and wall distance (normalized by the incoming boundary layer thickness), average and maximum $d^{+}$, and the total number of cells associated with each grid generated for the RAS domain are listed in Table 2. The hybrid RAS/LES grids were obtained by duplicating and mirroring the RAS grids which results in a cell count that is 4 times larger than those listed in Table 2. Several images of the grid are shown in Fig. 3. Topological nesting was used in several areas to help maintain a nearly constant grid spacing as the cross-sectional area of the domain varied, and a non-trivial topology was implemented in the vicinity of the fuel injectors to provide additional grid resolution for the smaller flow structures present when fuel is injected into the cavity. Also shown in this figure is the location of the recycling plane used to generate resolved turbulent content for the inflow of the scale-resolving simulations.

Table 2: Grid Attributes (RAS domain)

\begin{tabular}{||c|c|c|c||}
\hline \hline Attribute & Coarse & Medium & Fine \\
\hline \hline$\Delta / \delta$ & 0.063 & 0.042 & 0.028 \\
\hline$d_{1} / \delta$ & 0.0004 & 0.0004 & 0.0004 \\
\hline$d^{+}$(ave, max) & $0.50,0.85$ & $0.50,0.92$ & $0.50,0.99$ \\
\hline Total Cell Count & $2,540,336$ & $7,867,136$ & $24,585,120$ \\
\hline \hline
\end{tabular}

\section{Uncertainty Quantification}

Estimating model-form uncertainties is a challenging task requiring extensive validation efforts at conditions that are representative of the problem of interest. For many turbulent flows of engineering interest, the physical models chosen for the CFD simulation are often the dominant source of uncertainty. This is particularly true for turbulence closure models in a RAS framework. This uncertainty can (presumably) be reduced by resorting to a scale-resolving simulation approach like hybrid RAS/LES. Prior to assessing the model-form uncertainty, the numerical errors (i.e. grid convergence) should be quantified to ensure that the uncertainties associated with the numerical treatment are sufficiently small. This error source can be quantified using the Grid Convergence Index (GCI) ${ }^{14}$ The GCI is a grid convergence estimator derived from the generalized Richardson extrapolation formula and can be written as follows:

$$
\mathrm{GCI}=F_{s} \frac{\left|f_{1}-f_{2}\right|}{r^{p}-1}
$$

where $f$ is some flow parameter of interest evaluated at two different grid resolutions $\left(f_{1}\right.$ and $\left.f_{2}\right), r$ is the grid refinement ratio, and $p$ is order of accuracy of the numerical scheme. Finally, $F_{s}$ is a safety factor with recommended values taken to be either 3 (if the observed order of accuracy is assumed to be the theoretical value) or 1.25 (if the observed order of accuracy has been rigorously determined). It should be emphasized that the GCI, while based on Richardson Extrapolation, is not meant to be a "best estimate" of the numerical error. Instead, the intent is to provide a reasonable bound on the discretization error. Note that the impact of aleatoric (or random) uncertainties should also be considered prior to assessing the predictive accuracy of the relevant physical sub-models. However, the information required to formally perform this assessment was not provided in the documents that described the experiment.

The quantification of uncertainty is an even more daunting task for scale-resolving simulations due in part to the massive computational resources required to conduct each simulation. The level of statistical convergence must now be considered as a source of uncertainty, since the outputs of interest from an engineering perspective tend to involve time averages of the turbulent flow. The sub-grid models associated with LES, which explicitly vary with grid spacing, also blur the distinction between epistemic model-form errors and numerical resolution errors. These complications 

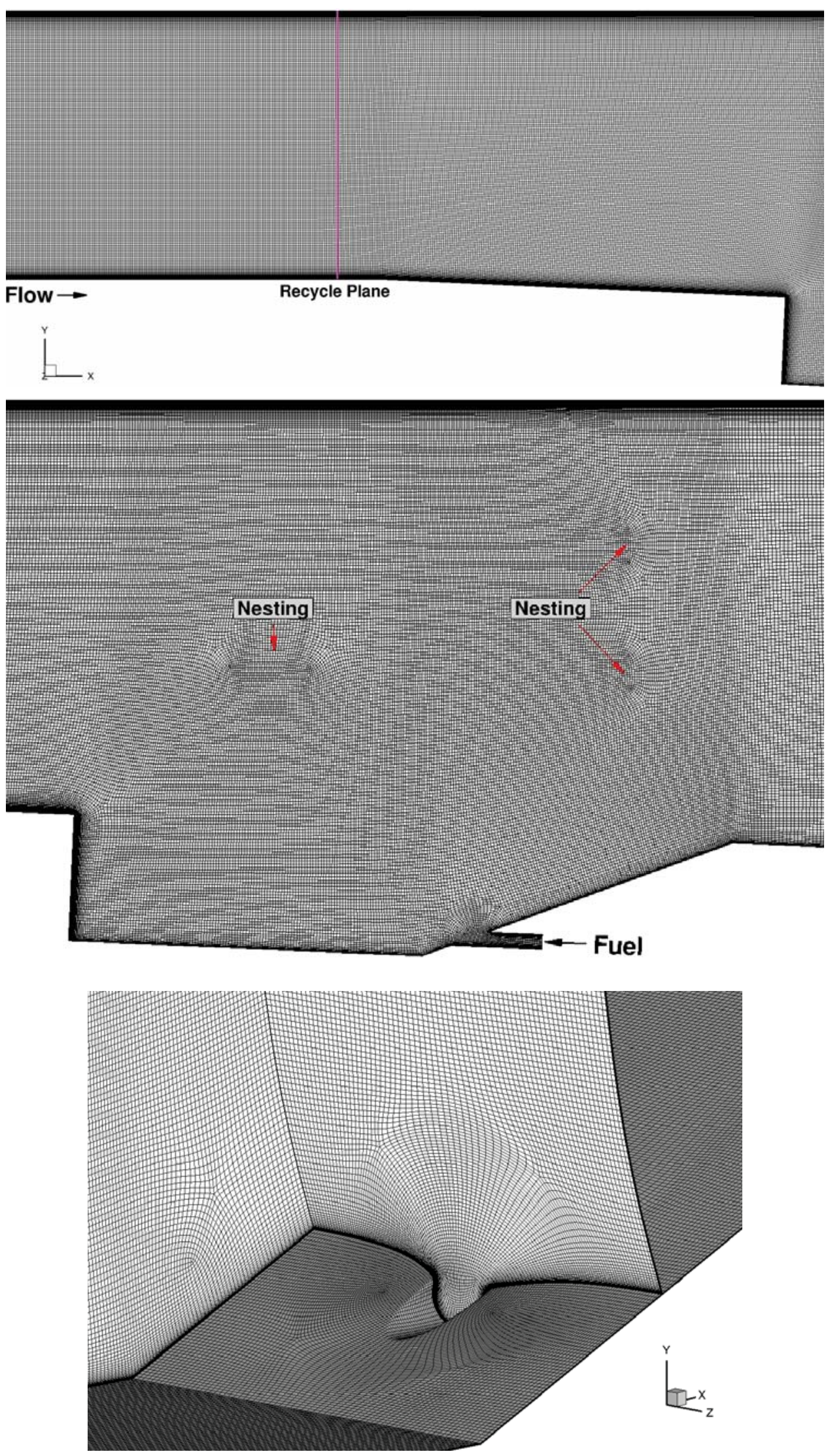

Figure 3: Grid images: overall view of the coarse grid (top 2 images), cavity fuel injection region (lower image) 
make any rigorous attempt at quantifying the numerical errors with LES or hybrid RAS/LES an arduous task that is seldom addressed in practice. In an attempt to simplify this process, the following viewpoint was adopted in this effort. The LES equations can be be written as the "under-resolved" Direct Numerical Simulation (DNS) equations plus (potentially) extra terms. The extra terms represent the modeled sub-grid contributions, which under normal circumstances, are explicitly dependent on the grid size. For instance, a typical molecular diffusion term in the DNS equation set

is replaced with a term like

$$
\text { Diffusion }=\mu \frac{\partial u_{i}}{\partial x_{j}}
$$

$$
\text { Diffusion }=\left(\mu+\mu_{s g s}\right) \frac{\partial u_{i}}{\partial x_{j}}
$$

in the modeled LES equations. The Sub-Grid Scale (SGS) viscosity, $\mu_{s g s}$, varies like $(\Delta x)^{2}$, so as the grid is refined towards that required by DNS resolution, the extra SGS terms approach zero like $(\Delta x)^{2}$. Based on this observation, one can choose to lump the SGS modeling error with the numerical error, which can in principle be estimated using measures like the GCI. In other words, the SGS terms can be decomposed as

$$
\mathrm{SGS}=\mathrm{SGS}_{\text {exact }}+\mathrm{SGS}_{\text {error }}
$$

where the $\mathrm{SGS}_{\text {exact }}$ is the SGS effect required to recover the DNS statistics (i.e. the ideal SGS model), and the SGS $_{\text {error }}$ can be considered as part of the numerical error. Note that an Implicit LES (ILES) utilizes precisely the same equation set as DNS. Hence, the SGS model error for an ILES (given the perspective described here) would be the leading error term of the numerical scheme.

While this viewpoint on uncertainty quantification for scale-resolving simulations is convenient, the practitioner must appreciate the limitations of this approach. The GCI is an error estimator derived from the Richardson extrapolation formula. This formula assumes that the dominant uncertainty in the solution is due to the smallest power of the grid spacing associated with the error of the numerical approach. This power is the minimum of 2 (assuming an explicit SGS model is used) or the leading error term of the truncated Taylor series associated with the numerical scheme. When this condition is satisfied, the numerical solution is said to be in its asymptotic convergence range, and the GCI can reliably be used to provide uncertainty bounds on the numerical error. However, if the grid is coarse enough such that higher-order terms are dominant, then the GCI estimate in general can not be relied upon to truly provide error bounds. However, from a practical viewpoint this scenario is often easy to detect. For instance, when the differences in solution estimates between grid levels are not monotonically reduced as the grid is refined, or when the difference in solution estimates are changing sign as the grid is refined. Hence, the informed user can often recognize this occurrence and tag the GCI value as unreliable, or add an additional safety factor if desired. At a minimum this approach at least provides a consistent means of documenting grid-related errors using a traceable framework.

\section{Reynolds-Averaged Results}

Reynolds-averaged simulations were performed for a variety of turbulence models to establish a baseline level of predictive accuracy for this flowfield based on the current state-of-the-art practices used for engineering purposes. These simulations were advanced in pseudo-time using an incomplete LU factorization scheme (with planar relaxation) ${ }^{15}$ with a Courant-Friedrichs-Lewy (CFL) number of 100 . The inviscid fluxes were evaluated using the LowDissipation Flux Split Scheme of Edwards ${ }^{16}$ with cell interface variable reconstruction achieved via the $\kappa=1 / 3$ Monotone Upstream-centered Scheme for Conservation Laws. The van Leer flux limiter ${ }^{17}$ was utilized to avoid spurious oscillations during this reconstruction process. The viscous fluxes were evaluated using $2^{\text {nd }}$-order accurate central differences with the constituent viscosities and conductivities computed from the polynomial fits of McBride. ${ }^{18,19}$ The turbulence models considered were the Menter-BSL $k-\omega$ model, ${ }^{20}$ Menter-SST $k$ - $\omega$ model, ${ }^{20}$ and the Gatski-Rumsey Explicit Algebraic Stress (Gatski-EAS) model. ${ }^{21}$ The turbulent Prandtl $\left(P r_{t}\right)$ and Schmidt $\left(S c_{t}\right)$ numbers, which control the turbulent transport of energy and mass, were chosen as 0.9 and 0.5 , respectively. Only simulations of Case 1 (no fuel injection) are discussed in this effort. Reacting and non-reacting simulations of the cases with fuel injection will be the focal point of follow-on investigations of this flowfield.

Solution convergence of the steady-state RAS was monitored by assessing the $\mathrm{L}_{2}$ norm of the equation set residual error, the mass flow error, and the surface friction force time histories. A sample convergence history is given in Fig. 4, which shows the normalized mass flow rate error and the $L_{2}$ norm of the steady-state residual error for the coarse grid Gatski-EAS simulation. At a minimum, the following iterative convergence statements were satisfied for each simulation: 
- The $\mathrm{L}_{2}$ norm of the steady-state residual error was reduced by at least 4 orders of magnitude.

- The surface friction force time history remained unchanged to 5 digits over the final 2500 iteration cycles.

- The relative mass flow rate error, $\left|\dot{m}_{\text {out }}-\dot{m}_{\text {in }}\right| / \dot{m}_{\text {in }}$, was less than $2.5 \times 10^{-7}$

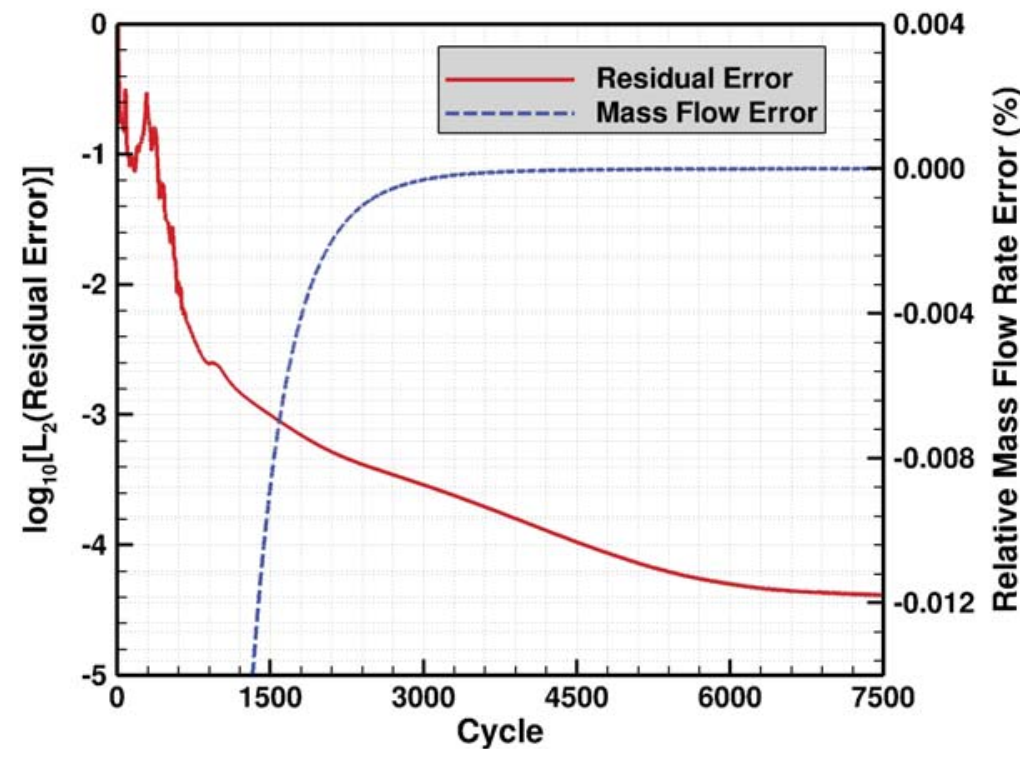

Figure 4: Typical steady-state iterative convergence history

Figure 5 shows the structure of the flow (via streamlines) inside the cavity flameholder as predicted by each of the turbulence models considered. Also shown are the streamlines extracted from time-averaged Particle Image Velocimetry (PIV) measurements taken by Tuttle et al. ${ }^{10}$ The predicted flow structure is qualitatively similar to the measurements with a dominant clockwise rotating recirculation zone and a smaller counter-clockwise rotating recirculation zone adjacent to the front wall of the cavity. From a flameholding perspective, the primary recirculation zone provides the mass exchange between the core flow and the hot cavity combustion products. The smaller recirculation zone simply enhances the cavity flow residence time. The most notable difference between the model predictions is the size and shape of the secondary recirculation zone associated with the Gatski-EAS model, which is larger and more elongated than those predicted by the Menter models. This difference is likely due to Reynolds stress anisotropies that EAS models (or more generally, non-linear eddy viscosity models) are capable of capturing. The impact of capturing this feature will become evident in the subsequent discussions of profile properties extracted at various locations within the cavity. Figure 6 shows the specific locations where flowfield profiles have been extracted.
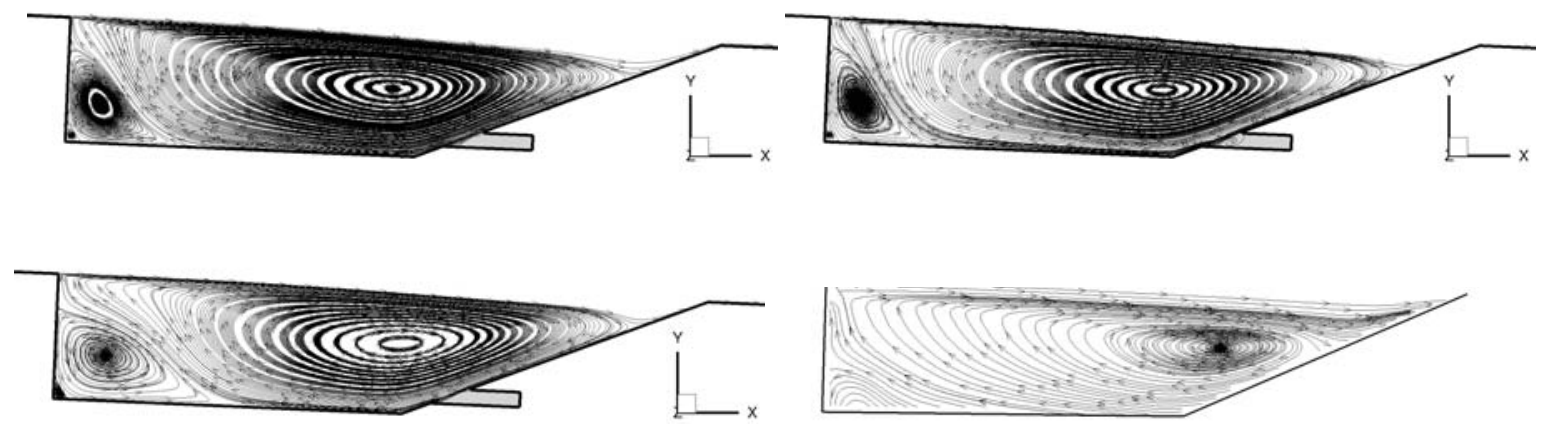

Figure 5: Streamlines extracted from measurements (bottom-right) and streamlines extracted from simulations: Menter-BSL (top left), Menter-SST (top-right), and Gatski-EAS (bottom-left) 


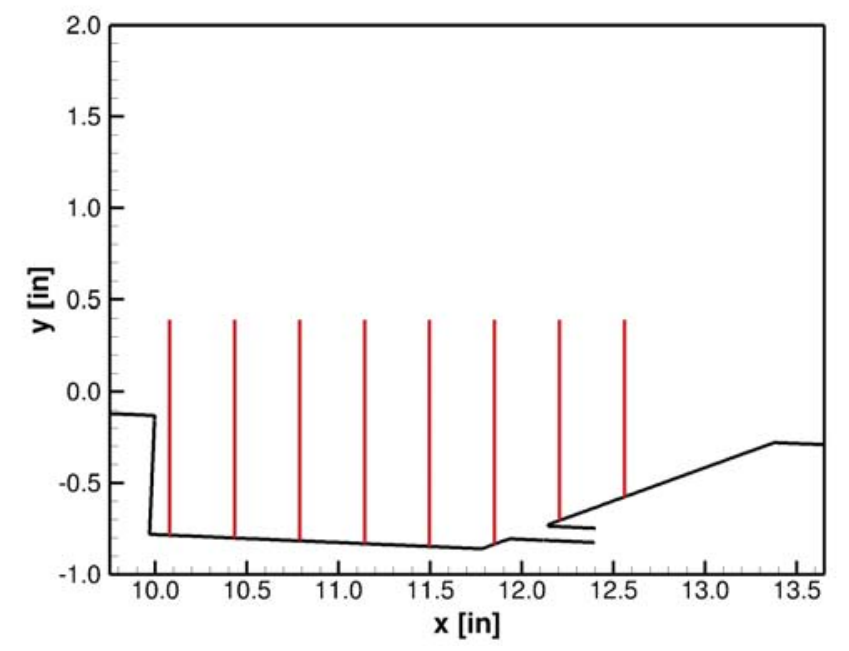

Figure 6: Locations within the cavity where profiles have been extracted

Prior to quantifying the predictive differences related to the choice of turbulence model, a formal solution verification study was performed to assess the adequacy of the grids used. Both qualitative (simple comparison of results on all 3 grids) and quantitative (via the GCI) assessments were performed and are shown in Fig. 7. The quantitative assessment based on the GCI is displayed as an error bar attached to the fine grid results, and should be interpreted as a bounding estimate of the error due to finite grid resolution. The error assessment was performed for each flow property of interest at every other profile station shown in Fig. 6. Only the analysis for the Gatski-EAS model simulations are shown in Fig. 7, since this model has the highest degree of non-linearity which typically leads to a larger sensitivity to grid resolution. The estimated numerical errors in the streamwise velocity profile are extremely small, suggesting that even the coarsest grid considered is sufficient to resolve this flow parameter. The errors extracted for the transverse (y-component) velocity predictions are more noticeable due to the small variation of the transverse velocity across the domain of interest. The largest errors for this quantity are located in the vicinity of the secondary recirculation zone (1st axial station shown). Finally, the only significant source of error associated with the $2^{\text {nd }}$-order velocity statistics is in the shear layer near the front of the cavity. In this region, the turbulence is in a non-equilibrium state as the flow transitions from a boundary layer to a free shear layer.

The solution sensitivity to the turbulence model is shown in the profiles displayed in Fig. 8. The selected streamwise stations are identical to those used for the numerical error assessment. The model-form uncertainty is substantial, which was expected given that the region of interest is a large separated flowfield. Focusing first on the streamwise velocity profiles, it is clear that the Gatski-EAS model is predicting a slower shear spreading rate than that predicted by the Menter models. The peak reverse flow value (near the lower wall of the cavity) given by the Gatski-EAS model is also noticeably smaller. The significant disparity in the transverse velocity at the $x=10.43$ inch station is a result of the differences in the secondary recirculation zone structure noted in Fig. 5. This flow feature was significantly larger in the Gatski-EAS results, which explains the smaller velocities (in magnitude) noted near the cavity floor at this station. The transverse velocity values obtained from all 3 models are in closer agreement at the $x=11.14$ inch station, but the profiles again show appreciable differences further downstream. Relatively small deviations in the shear layer spreading rate and/or deflection angle will alter how the shear layer bifurcates at the re-attachment location along the aft-wall of the cavity. These details (which are small in velocity magnitude) are more pronounced when visualizing the transverse velocity profiles. The bottom two rows of images compare the turbulence kinetic energy and dominant shear stress (displayed as a covariance) profiles. In general, the Menter-SST model predicts values for these properties that are less than or equal to (in magnitude) those produced by the Menter-BSL model. This trend is to be expected since the only difference between these models is the stress limiter present in the SST variant; a feature that limits the turbulence levels relative to the BSL model. The Gatski-EAS results show an even greater reduction in the turbulence levels within the cavity. It should be noted that algebraic stress models tend to have implied built-in stress limiters (relative to their linear counterparts). ${ }^{22}$ However, the implied shear stress limiting effect associated with the Gatski-EAS model is somewhat less than that explicitly present in the Menter-SST model. Hence, some of the reduction in the turbulence predicted by the Gatski-EAS model must be due to the anisotropy in the normal stress 

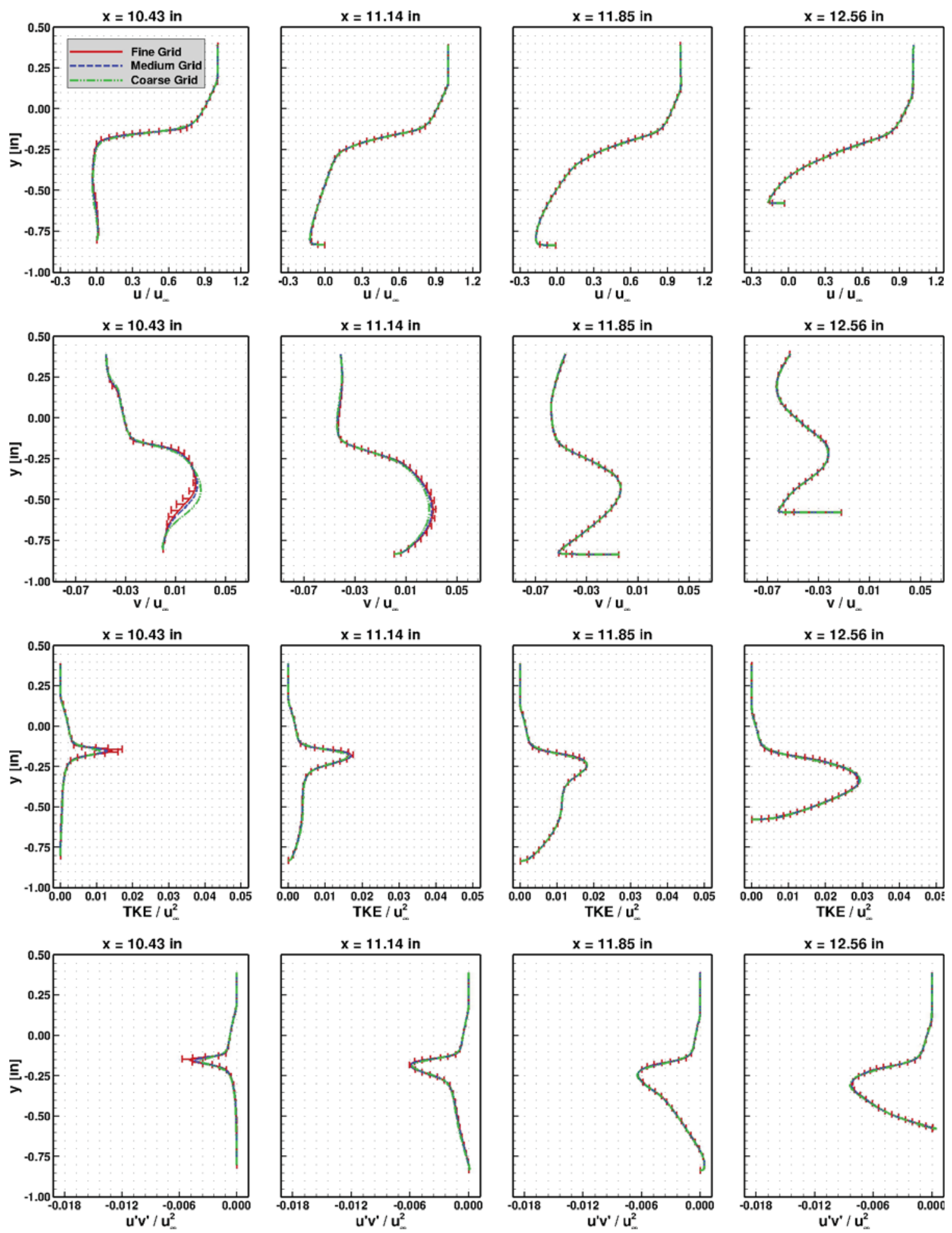

Figure 7: Velocity components, turbulence kinetic energy, and x,y velocity covariance profile comparisons on each grid (error bars show the GCI on the fine grid results) 
components predicted by this model.

The velocity statistics (mean and $2^{\text {nd }}$-order correlations) are compared with the PIV measurements in Fig. 9. The mean streamwise velocity comparisons show that both of the Menter models are predicting the proper spreading rate of the shear layer. The Gatski-EAS model predictions are also reasonable, but the spreading rate is somewhat slower than the measurements indicate. The fact that the spreading rates were predicted correctly (or slightly underpredicted) was not expected. Standard RAS models (i.e. models without compressibility corrections) are known to overpredict the spreading rates of shear layers with a high convective Mach number. The present flow has a convective Mach number of 0.9 , which is large enough to expect compressibility effects to be significant. Presumably, the short cavity length (relative to the thickness of the approach boundary layer) has limited the impact of this compressibility feature. It should also be noted that the simulations predict a core flow velocity that is systematically larger (by approximately $10 \%)$ than the PIV measurements. As pointed out in Ref. 13, this may be a consequence of how the flow was seeded for the PIV measurements. The flow was seeded in the boundary layer upstream of the cavity through an angled slot injector. The flow rate of the seeded air was intentionally kept small (10 SLPM) to minimize disturbances to the flow. Given the thick $(\approx 0.25$ inches) approach boundary layer, it is quite possible that the outer portion of the boundary layer may be insufficiently seeded. The net result would be a measurement that is biased towards lower velocity values.

Overall, the transverse velocity values compare well with the measurements (particularly the Gatski-EAS results) with the most notable exception being the transverse velocity values in the shear layer at some of the downstream axial stations. The values within the shear layer are well predicted initially, but start to deviate from the measurements at the 10.79 inch station, and this trend progressively worsens at stations further downstream. Note also the difference in the predictions within the cavity at the $x=10.43$ inch station. As pointed out in the discussion of the flow structure from Fig. 5, the Gatski-EAS model predicted a secondary recirculation zone that was notably different than what was predicted by the Menter models. The favorable agreement between the Gatski-EAS predictions and the measurements at this station suggest that this flow structure is a closer representation of what was measured.

The velocity variances and covariance comparisons show a strong dependence on the turbulence model. With few exceptions, the Gatski-EAS model produced results that were closer to the measured values. All of the models predicted the streamwise normal stresses reasonably well, but both of the Menter models consistently overpredicted the transverse normal stress component by a significant margin. The Gatski-EAS model allows for Reynolds stress anisotropies (a feature not captured by linear eddy viscosity models). Hence, this model is able to predict normal stress components that are substantially different from one another. The Reynolds shear stress profiles are also substantially better predicted by the Gatski-EAS model. The Menter-SST shear stress values are considerably smaller (in magnitude) than the Menter-BSL predictions (a direct consequence of the stress limiter in the SST model), but they are still in excess of the measurements by at least a factor of 2 . As a final note, the measured velocity variances are not asymptoting to zero as the freestream is approached. As pointed out previously in the discussion of the mean velocity values, the PIV seed particle count may be insufficient in the outer portion of the shear layer. The fact that the measured variance values are not decreasing as the freestream is approached appears to support this hypothesis. If this issue is indeed responsible for a deficit in the measured mean velocity values (a deficit as large as 10\%), then one would expect even larger errors in the higher-order statistics.

\section{Hybrid RAS/LES Formulation}

The hybrid RAS/LES methodology used in this effort is based on the framework originally developed in Ref. 2, with subsequent variants described in Refs. 23 and 24. This framework is designed to enforce a RAS behavior near solid surfaces, and switch to an LES behavior in the outer portion of the boundary layer and free shear regions. Hence, this formulation can be thought of as a wall-modeled LES approach, where a RAS closure is used as the near-wall model. The basic idea is to blend the RAS eddy viscosity value with the LES SGS viscosity, along with any transport equation that involves a common RAS and SGS property. In this effort, the Menter-BSL $k-\omega$ RAS model ${ }^{20}$ was blended with the one-equation SGS model of Yoshizawa. ${ }^{25}$ The Yoshizawa model involves an evolution equation for the SGS turbulence kinetic energy, hence the blended expressions that are appropriate for this model combination are:

$$
\begin{aligned}
\text { Hybrid RAS/SGS viscosity } & =(F)[\text { RAS viscosity }]+(1-F)[\text { SGS viscosity }] \\
\text { Hybrid RAS/SGS k-equation } & =(F)[\text { RAS k-equation }]+(1-F)[\text { SGS k-equation }]
\end{aligned}
$$

where $F$ is a blending function that varies between 0 and 1 . Note that the transport equation for the RAS specific dissipation rate $(\omega)$ does not have an SGS counterpart. Hence, the blending is not applied to this equation, and all of the terms in this equation that involve the eddy viscosity are evaluated based on the RAS relationships.

The motivation behind the development of this particular hybrid RAS/LES framework is two-fold. First, the 

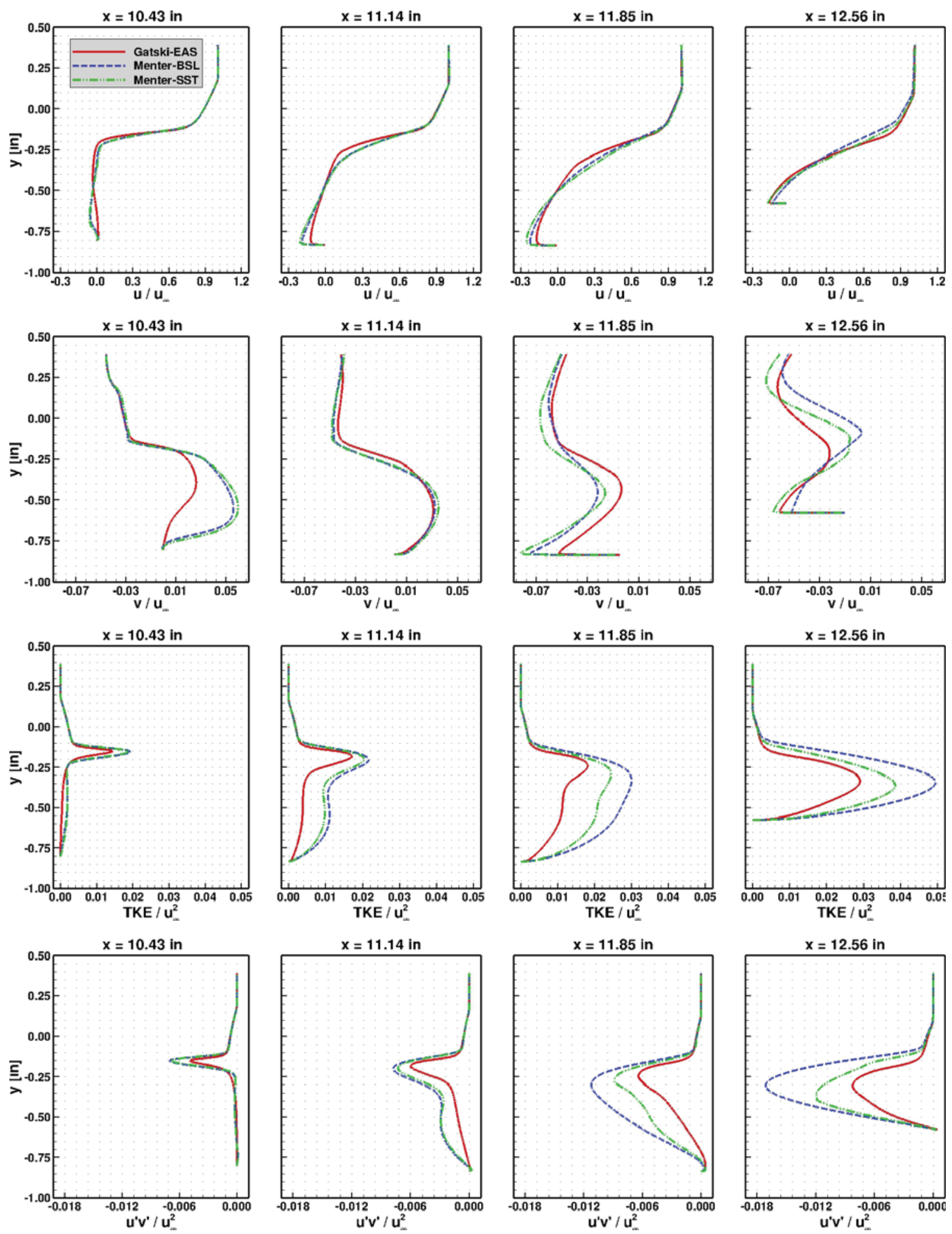

Figure 8: Velocity components, turbulence kinetic energy, and $\mathrm{x}, \mathrm{y}$ velocity covariance profiles comparisons for each turbulence model 

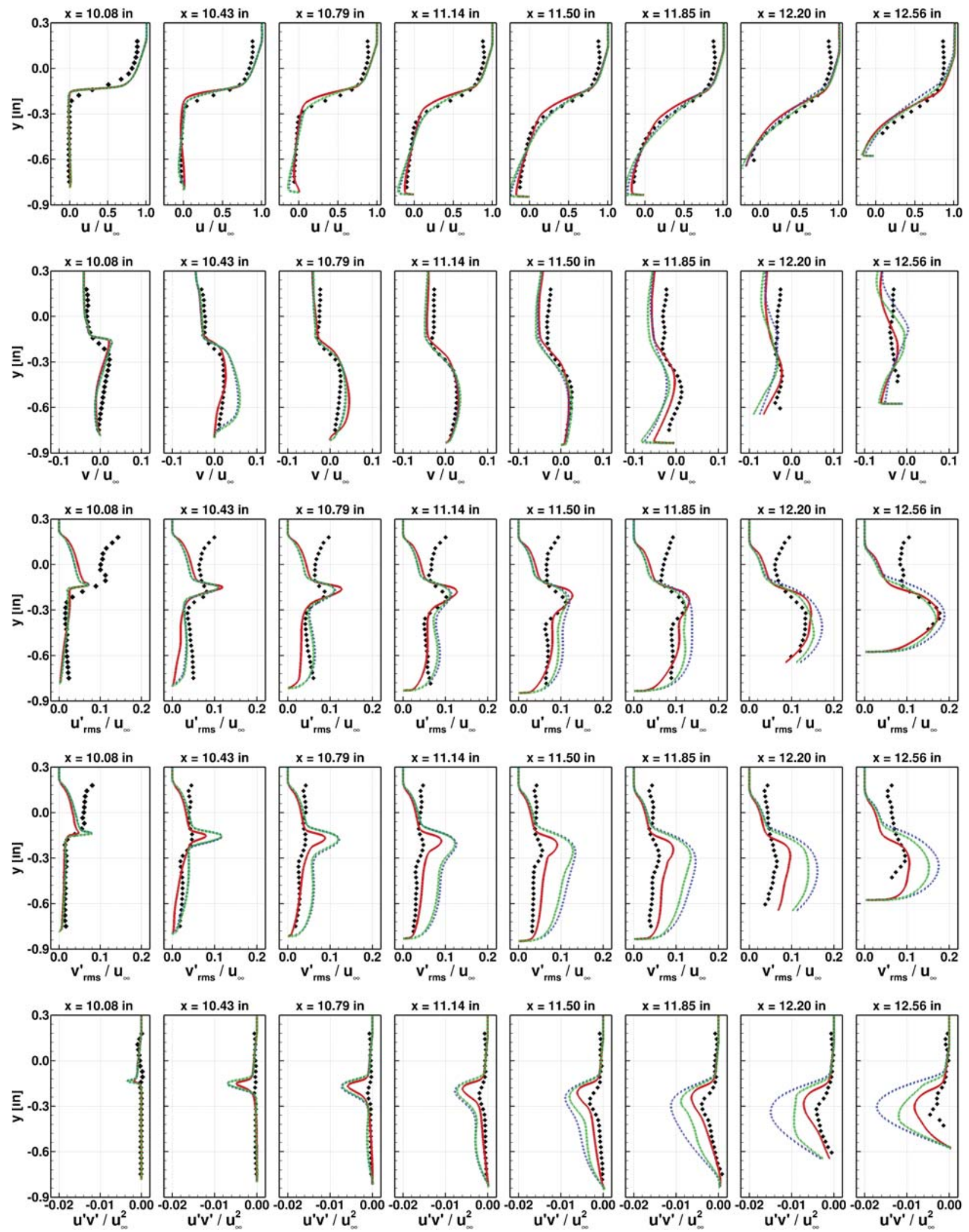

Figure 9: Comparison of fine grid RAS predictions with measurements: PIV measurements (symbols), Gatski-EAS (solid red lines), Menter-BSL (dashed blue lines), Menter-SST (dash-dot green lines) 
blending of two independent RAS and LES closure models offers the flexibility of having an optimized set of closure equations for both RAS and LES modes. The second (and more critical) driving factor was the desire to alleviate the difficulties associated with the design of grid topologies that are appropriate for purely grid-dependent blending paradigms such as those utilized by the original Detached Eddy Simulation (DES) ${ }^{26,27}$ methodology. The movement away from simple grid-dependent blending strategies has become more prevalent in recent years. ${ }^{3,4}$ In fact, even the DES formulation has evolved ${ }^{4,28}$ to incorporate flow-dependent functions to manage the RAS and LES regimes.

The particular blending function $(F)$ used in this effort is parameterized by the ratio of the wall distance $d$ to a modeled form of the Taylor microscale $(\lambda)$ :

$$
\begin{aligned}
& F=\frac{1}{2}\left\{1-\tanh \left[5\left(\frac{\kappa}{\sqrt{C_{\mu}}} \eta^{2}-1\right)-\tanh ^{-1}(0.98)\right]\right\} \\
& \eta=\frac{d}{\alpha \lambda}, \quad \lambda=\sqrt{\frac{\mu}{C_{\mu} \rho \omega}}
\end{aligned}
$$

where $\kappa$ is the von Karman constant $(0.41), C_{\mu}$ is $0.09, \alpha$ is a user-defined model constant, and the factor $\tanh ^{-1}(0.98)$ is used to force the balancing position of $F$ (i.e. the position where $\kappa \eta^{2}=\sqrt{C_{\mu}}$ ) to 0.99 . The value chosen for $\alpha$ provides control over the $d^{+}$position where the average LES to RAS transition point (defined as $F=0.99$ ) occurs. If resolved LES content is desired for an attached boundary layer, then this constant should be set such that the transition point occurs in the region where the boundary layer wake law starts to deviate from the log law. If the transition point is enforced at a lower $d^{+}$value that is well within the log law region, a dual log layer appears (an effect sometimes referred to as the log layer mismatch). ${ }^{23,29}$ Conversely, if the transition point is enforced at a $d^{+}$value that extends well into the wake region, then the level of resolved turbulent content will be reduced. Details on a procedure to analytically determine the value for $\alpha$ that corresponds to a target $d^{+}$value is described in Ref. 23 .

\section{Low-Dissipation Numerical Formulation}

The low-dissipation numerical framework utilized by the cell-centered finite volume VULCAN-CFD flow solver relies on the blending of a non-dissipative advection operator $\left(2^{\text {nd }}, 4^{\text {th }}\right.$, or $6^{\text {th }}$ order $)$ with a dissipative upwind-biased operator. The dissipative advection operators available in the solver include the MUSCL ${ }^{17}$ ( $2^{\text {nd }}$ or $3^{\text {rd }}$ order), PPM ${ }^{30}$

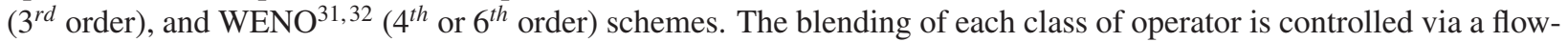
dependent function that attempts to discriminate between inviscid and viscous dominated flow features. A variety of sensors have been proposed in the literature for this purpose, two of which are considered here. The Ducros ${ }^{33}$ sensor distinguishes each flow regime by comparing the magnitude of the vorticity (viscous flow marker) with the velocity divergence (inviscid flow marker) via the following functional form:

$$
\psi=\frac{(\vec{\nabla} \cdot \vec{V})^{2}}{(\vec{\nabla} \cdot \vec{V})^{2}+(\vec{\nabla} \times \vec{V})^{2}+\epsilon^{2}}
$$

where $\epsilon$, a small number to prevent division by zero in uniform flow regions, is defined as

$$
\epsilon=\frac{1 \times 10^{-8} V_{\infty}}{\max \left(\bar{\triangle}_{x}, \bar{\triangle}_{y}, \bar{\Delta}_{z}\right)}
$$

The Larsson ${ }^{34}$ sensor utilizes the heavy-side function to define a binary (on/off) sensor,

$$
\psi= \begin{cases}0, & \theta \leq 1 \\ 1, & \theta>1\end{cases}
$$

where the argument $\theta$ is given by

$$
\theta=\frac{-(\vec{\nabla} \cdot \vec{V})}{\max \left(C_{1}|\vec{\nabla} \times \vec{V}|, C_{2}[c / \sqrt[3]{V o l}]\right)}
$$

This sensor retains the sign on the velocity divergence, so it distinguishes between compression and expansion processes. A positive velocity divergence value (expansion process) always returns a zero value. Hence, a judicious 
selection of the $C_{1}$ and $C_{2}$ coefficients can lead to a sensor that serves as a shock detector if this is the behavior that is desired. An alternative definition of $\theta$ was also considered in this effort that ignores the sign of the velocity divergence, i.e.

$$
\theta=\frac{(|\vec{\nabla} \cdot \vec{V}|)}{\max \left(C_{1}|\vec{\nabla} \times \vec{V}|, C_{2}[c / \sqrt[3]{V o l}]\right)}
$$

This sensor does not distinguish between expansions and compressions (so it is similar to the Ducros sensor from that perspective), while still offering some level of fine-grained control through the selection of the $C_{1}$ and $C_{2}$ coefficients. This sensor will be termed the modified Larsson sensor in subsequent sections. Further sensor implementation details (smoothing, boundary treatment, etc.) are described in Ref. 8 along with a more detailed description of the dissipative and non-dissipative advection schemes.

\section{Hybrid Reynolds-Averaged / Large Eddy Simulation Results}

The hybrid RAS/LES utilized the low-dissipation numerical framework described previously to encourage the development of resolved turbulent content. Both the MUSCL $(\kappa=1 / 3)$ and WENO schemes were considered for the dissipative advection operator, and the $4^{\text {th }}$-order symmetric reconstruction approach was chosen for the non-dissipative scheme. The flux limiter used for the MUSCL scheme was the UNO limiter of Suresh and Huynh, ${ }^{35}$ which can be regarded as a second-order extension of the minmod TVD limiter. The viscous fluxes were evaluated in the same manner as that used for the Reynolds-averaged simulations described in the previous section. All of the time-accurate hybrid RAS/LES solutions were advanced in time using a dual time-stepping approach that combined a Diagonalized Approximate Factorization (DAF) scheme ${ }^{36}$ for integration in pseudo-time, with a 3-point backwards finite difference approximation for integration in real-time. The values selected for the physical time-step and sub-iteration CFL constraint were $0.05 \mu s$ and 10.0, respectively. The time-step was chosen based on cell residence time considerations to ensure that turbulent structures would traverse less than one grid cell length per time-step (i.e. CFL $<1$ ). The sub-iteration process was carried out until the residual error dropped 2 orders of magnitude. This level of convergence typically required 7-10 sub-iterations for each physical time-step. The value specified for the parameter $\alpha$ that appears in the hybrid RAS/LES blending function (see Eq. 6) was 39.1. This value was determined using the analytical procedure described in Ref. 23.

In order to provide an inflow condition with resolved turbulent content, a recycling/rescaling procedure ${ }^{37}$ was employed. The particular strategy used here can be described as follows:

- The velocity, temperature, and density fluctuations are extracted from the desired recycling station (see Figs. 1 and 3) along with the transported turbulence variables (i.e. $k$ and $\omega$ ).

- The extracted flow properties are rescaled based on boundary layer scaling laws.

- The rescaled fluctuations are added to the mean RAS inflow profile (see Fig. 2), and the rescaled instantaneous turbulence variables replace their RAS counterparts.

- An intermittency function is utilized to prevent recycled fluctuations from corrupting the freestream region.

- A random spanwise shift procedure is used to mitigate the tendency of the large scale structures to persist within a fixed spanwise path by the recycling process.

Further details associated with this procedure are documented elsewhere. ${ }^{23,24}$

The initial state of the hybrid RAS/LES flowfield was defined using the converged RAS results. Moreover, artificial fluctuations were added to the approach boundary layer flow to reduce the start-up time required to generate resolved turbulent content. The simulations were monitored as a function of time to assess the establishment of a statistically stationary state prior to gathering flowfield statistics (or analyzing any instantaneous flow properties). Particular emphasis was given to the mass flow error and the integrated friction force time histories. A sample time history is shown in Fig. 10. Based on these metrics, a statistically stationary state was deemed to be established after roughly 25,000 time-steps. This integration period corresponded to 10.6 flow-through times, defined as the time required for the freestream particles to traverse the length of the cavity. After this time period, flowfield statistics were gathered for at least another 75,000 time-steps (31.8 flow-through times).

The low dissipation numerical framework utilizes a blending strategy to control where the dissipative and nondissipative operators are invoked. In principle, the non-dissipative operators are desired wherever resolved turbulent content is present (boundary layers, free shear layers, etc.) and the dissipative operators are desired in regions where 


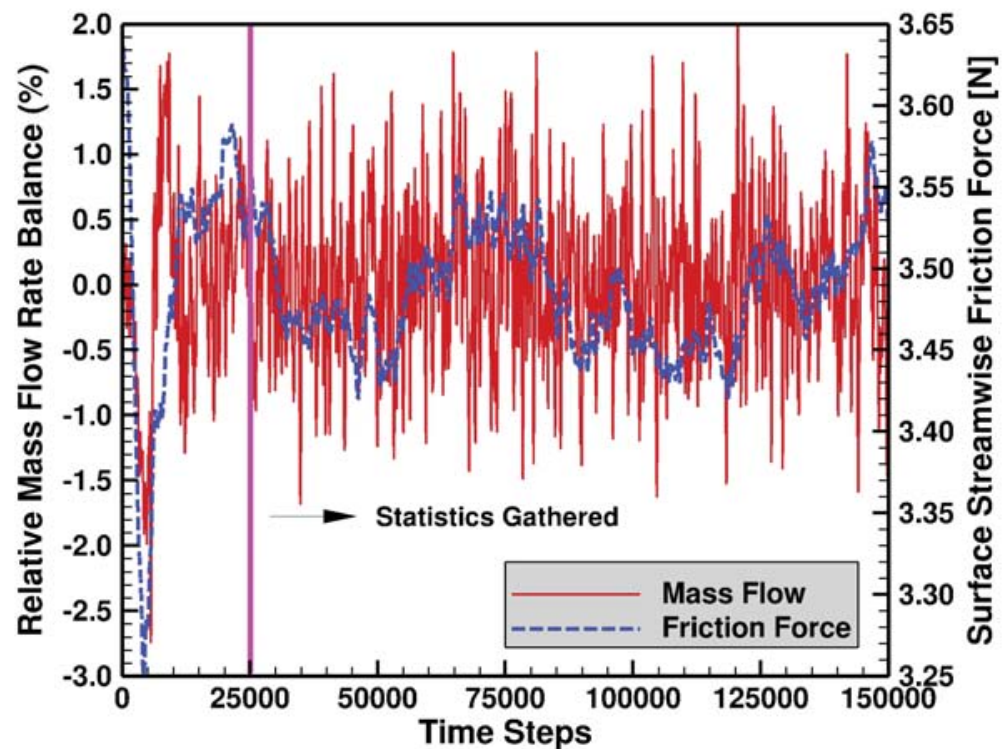

Figure 10: Typical hybrid RAS/LES time history used to assess statistical stationarity

inviscid flow features are dominant (particularly near shock waves). The three blending sensors described previously were evaluated for this purpose. An instantaneous snapshot of each sensor is shown in Fig. 11. The Ducros ${ }^{33}$ sensor is shown in the top image. This sensor simply compares the magnitude of vorticity with the absolute value of the velocity divergence, and is a popular choice used in the research community. ${ }^{12,23}$ This sensor is tagging most of the inviscid portions of the flowfield appropriately. However, it is also tagging a significant fraction of the viscousdominated flow regime (particularly inside the cavity) as a region to be treated by the dissipative operators. The random manner in which this sensor is being activated in regions where resolved turbulent structure is present is also an undesirable feature. The Larsson ${ }^{34}$ sensor, with the $C_{1}$ and $C_{2}$ coefficients tuned to detect significant compression waves (5.0 and 0.05, respectively), is shown in the middle image of Fig. 11. This blending strategy only invokes the dissipative numerical scheme where it is absolutely necessary for stability. However, it is prone to exhibit nonphysical fluctuations in the inviscid regions of the flow (where there is limited natural viscous effects to provide sufficient dissipation). The $C_{1}$ and $C_{2}$ coefficients can be altered to force more of the inviscid region to be tagged for the dissipative algorithm, but this sensor is sensitive to the sign of the velocity divergence. Hence, regions of flow expansions are inevitably going to be tagged as a non-dissipative flow region. This issue is overcome by desensitizing the Larsson sensor to compressions and expansions. The results from this modified form of the Larsson sensor is shown in the bottom image of Fig. 11. The behavior of this sensor (with $C_{1}=5.0$ and $C_{2}=1.0 \times 10^{-8}$ ) is qualitatively similar to that of Ducros, but with its undesirable features minimized. Based on these observations, the modified Larsson sensor was selected for all of the hybrid RAS/LES results described below.

A comparison of time-averaged velocity profiles in wall units is given in Fig. 12. The van Driest II transformation was used in the post-processing of the computational results shown in this figure to account for compressibility effects. Also shown in this image is the hybrid RAS/LES blending function (Eq. 6) to illustrate the location in the boundary layer where the simulation transitions (in a time-averaged sense) from RAS to LES. The purpose of this comparison is to ensure that the value chosen for $\alpha$ is appropriate, and that the recycling process recovers the proper boundary layer scaling behavior. A comparison of the hybrid RAS/LES result with the RAS profile shows evidence of a slight $\log$ layer mismatch just before the start of the wake region, but overall the profile matches the RAS result reasonably well (confirming that a feasible value has been chosen for $\alpha$ ). There is some deviation from the theoretical log law result that used a value of 0.40 for $\kappa$ with B set to 5.1. However, the deviation appears in both the RAS and hybrid RAS/LES results, so it is not a result of the recycling process.

Instantaneous images of the density gradient magnitude are shown for the coarse, medium, and fine grids in Fig. 13. This quantity is similar to a Schlieren image and allows both turbulent structures and inviscid structures (shock waves and expansions) to be visualized. As evident in the images shown, even the data extracted from the coarsest grid displays a rich array of flow structures, which includes weak eddy shocklets emanating from the movement of large 


\section{Sensor: 0.00 .10 .20 .30 .40 .50 .60 .70 .80 .91 .0}
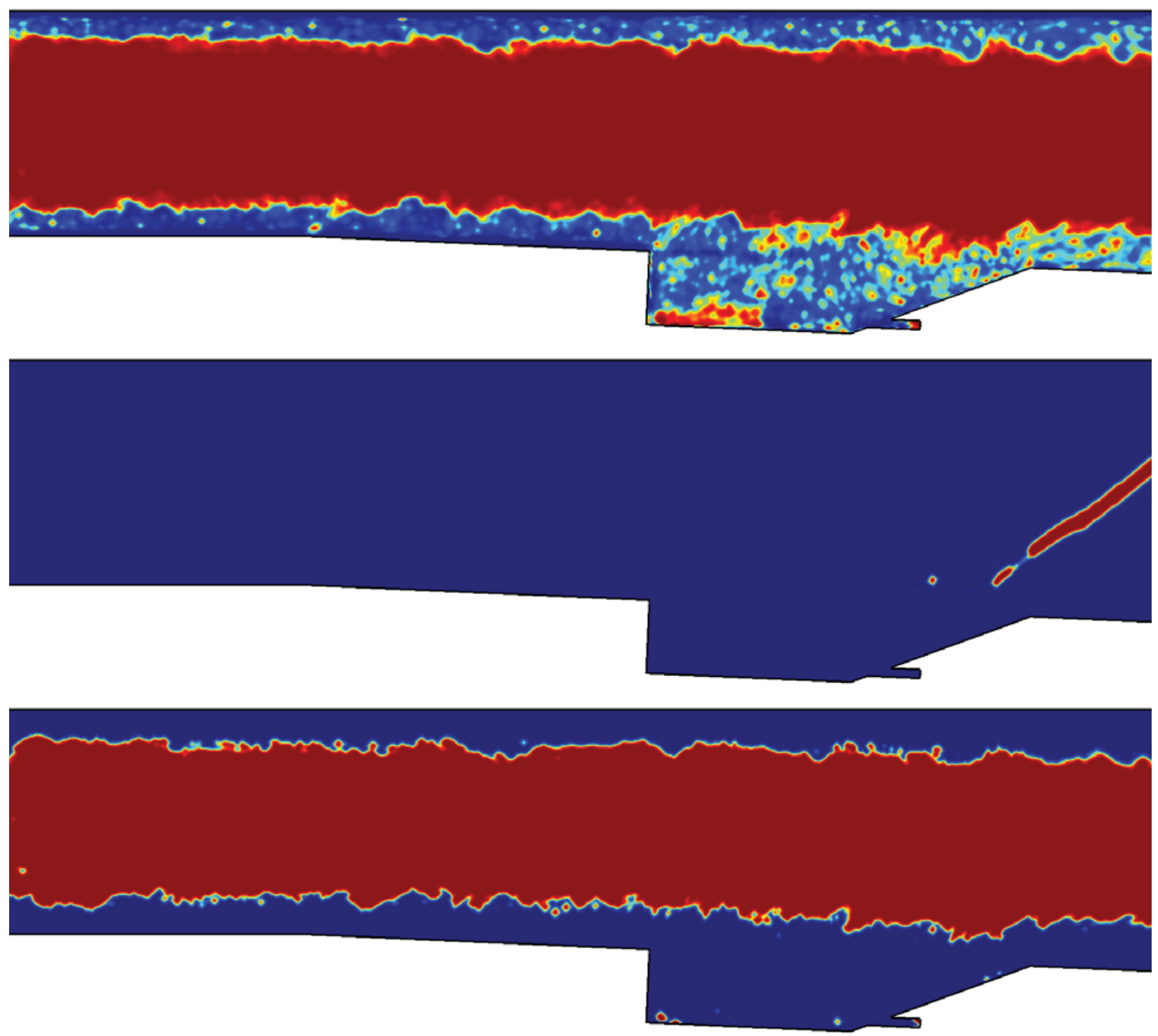

Figure 11: Comparison of sensor formulations controlling the blend of dissipative and non-dissipative schemes: Ducros (top), Larsson (middle), modified Larsson (bottom)

coherent structures in the shear layer and a crisp shear layer re-attachment shock system near the back face of the flameholder. All of the grid levels show evidence of the same qualitative flow features (i.e. no major feature is missing as the grid is coarsened), but the finest grid clearly shows a wider range of scales being resolved.

Figure 14 shows the structure of the time-averaged flow inside the cavity flameholder obtained from the hybrid RAS/LES model. The result shown was extracted from the fine grid solution that used the MUSCL scheme with the UNO limiter as the dissipative portion of the hybrid numerical scheme. Solutions were also obtained with the $4^{\text {th }}$-order WENO scheme used instead of the MUSCL approach. However, the differences in the predictions were found to be much smaller than the variability noted with grid resolution (to be discussed later), so these results are omitted for brevity. For comparison purposes, the steady-state Gatski-EAS RAS result from Fig. 5 has been repeated here, since this model proved to be the best performer of the RAS models considered. The hybrid RAS/LES cavity flow structure shows a secondary recirculation zone that is almost as large as that predicted by the Gatski-EAS model. This was an 


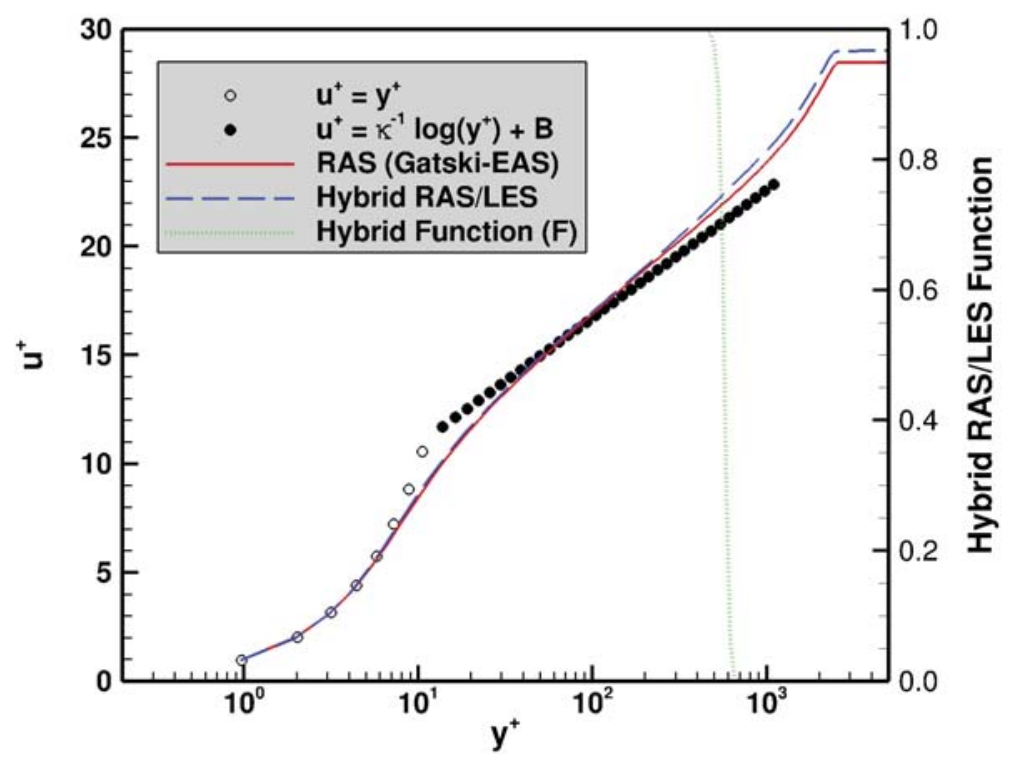

Figure 12: Comparison of the hybrid RAS/LES approach boundary layer profile with RAS and log law theory

important flow feature noted in the Gatski-EAS results when comparisons were made with measurements. The size and the shape of the primary recirculation zones of both predictions are similar as well.

Figure 15 shows the variation of several statistics involving the velocity components as the number of samples is increased. Each time-step (after a statistically stationary state was obtained) contributed to the sample size, so larger sample sizes imply an integration further in time. The profiles shown include the mean streamwise and transverse velocity components, turbulence kinetic energy (both the resolved and SGS components), and the resolved and SGS portions of the $\mathrm{x}, \mathrm{y}$ velocity covariance. The profile statistics show very little variation suggesting that 31.8 flowthrough times $(75,000$ time-steps) was sufficient to converge the statistics. The largest statistical variability appears in the mean transverse velocity predictions, and it is less than $0.4 \%$ of the freestream velocity. Note that measures of the statistical variability can be misleading if the total integration time is far from being sufficient to have captured several periods of the largest physical time scales associated with the flow. An analysis of the autocorrelation of the fluctuating velocity field $\left(R_{i i}=\overline{u_{i}^{\prime}(t) u_{i}^{\prime}(t+\tau)}\right)$ can provide information in this regard. The autocorrelation (normalized by the local turbulence kinetic energy value) was evaluated at 3 strategic locations:

- A location in the wake region of the approach boundary layer

- A location within the shear layer that spans the cavity length

- A location close to the center of the time-averaged primary recirculation zone

and the results are shown in the left image of Fig. 16. The data used to generate this image covers 10.6 cavity flow-through times ( $1 / 3$ of the integration time used to gather statistics), and were extracted from the coarse grid simulations. By examining the autocorrelations, it is clear that the integration time is sufficient for the boundary and shear layer regions of the flow (i.e. the velocity fluctuations become decorrelated after roughly $20 \mu s$ ). However, the integration time shown is not sufficient for the region near the center of the primary recirculation zone, since the velocity fluctuations are not yet decorrelated after $625 \mu \mathrm{s}$. The largest physically relevant time scale (integral time scale) at each location considered can be obtained by integrating the area under the autocorrelation curves. ${ }^{38}$ If the total integration time is large relative to the integral time scale, then an estimate for the convergence of the statistics can be obtained from a finite sample size using the approach outlined in Ref. 38. The autocorrelation data was gathered at only a few select points in this effort, so quantified values could not be established for the profile data shown in Fig. 15. However, given the general behavior of the points selected, the total integration time used to gather the statistics is expected to be sufficient except for the immediate vicinity near the core of the recirculation zones (where the mean velocity is extremely small).

The autocorrelations can also provide information about the range of scales present in the simulation. Two different points in the wake region of the approach boundary layer (one near the recycling plane and one just before the cavity 

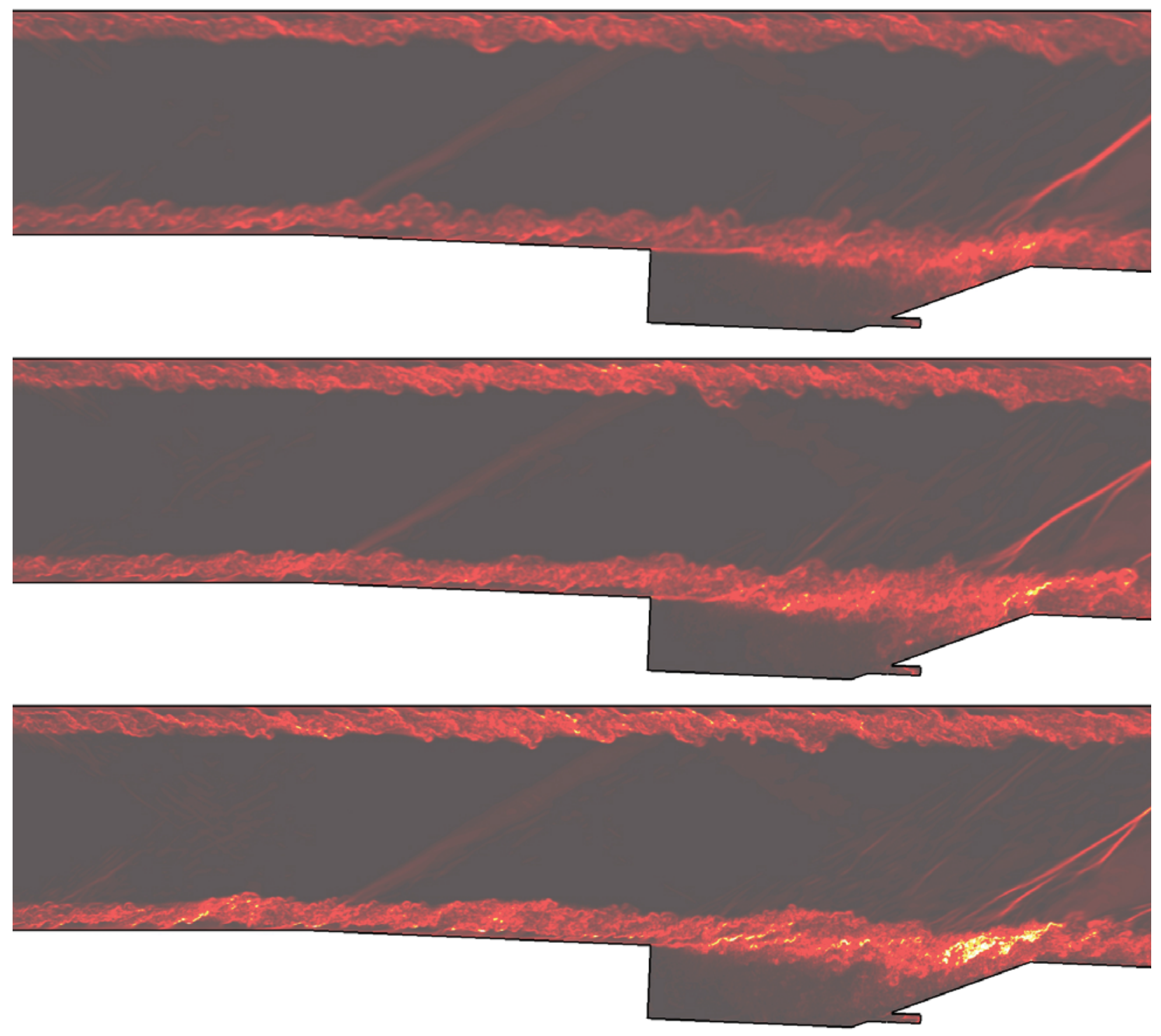

Figure 13: Hybrid RAS/LES instantaneous images of density gradient magnitude: coarse grid (top), medium grid (middle), fine grid (bottom)
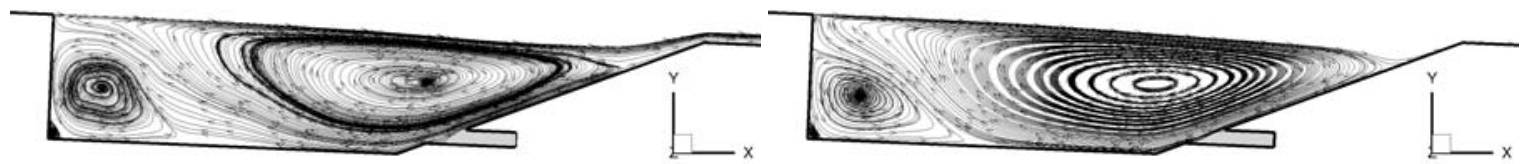

Figure 14: Streamlines extracted from simulations: hybrid RAS/LES (left), RAS with Gatski-EAS (right) 

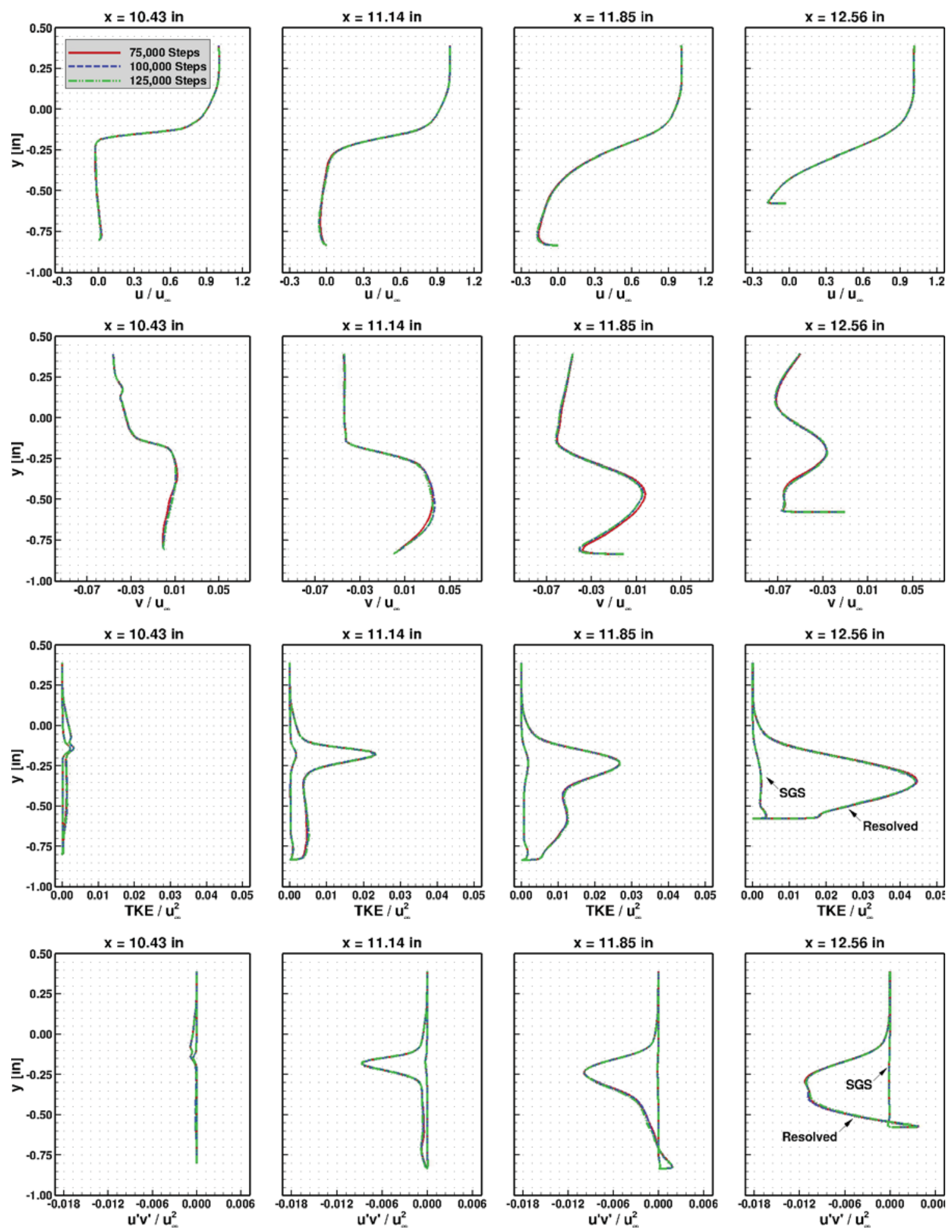

Figure 15: Velocity components, turbulence kinetic energy, and $\mathrm{x}, \mathrm{y}$ velocity covariance profile comparisons with increasing statistical sample size 

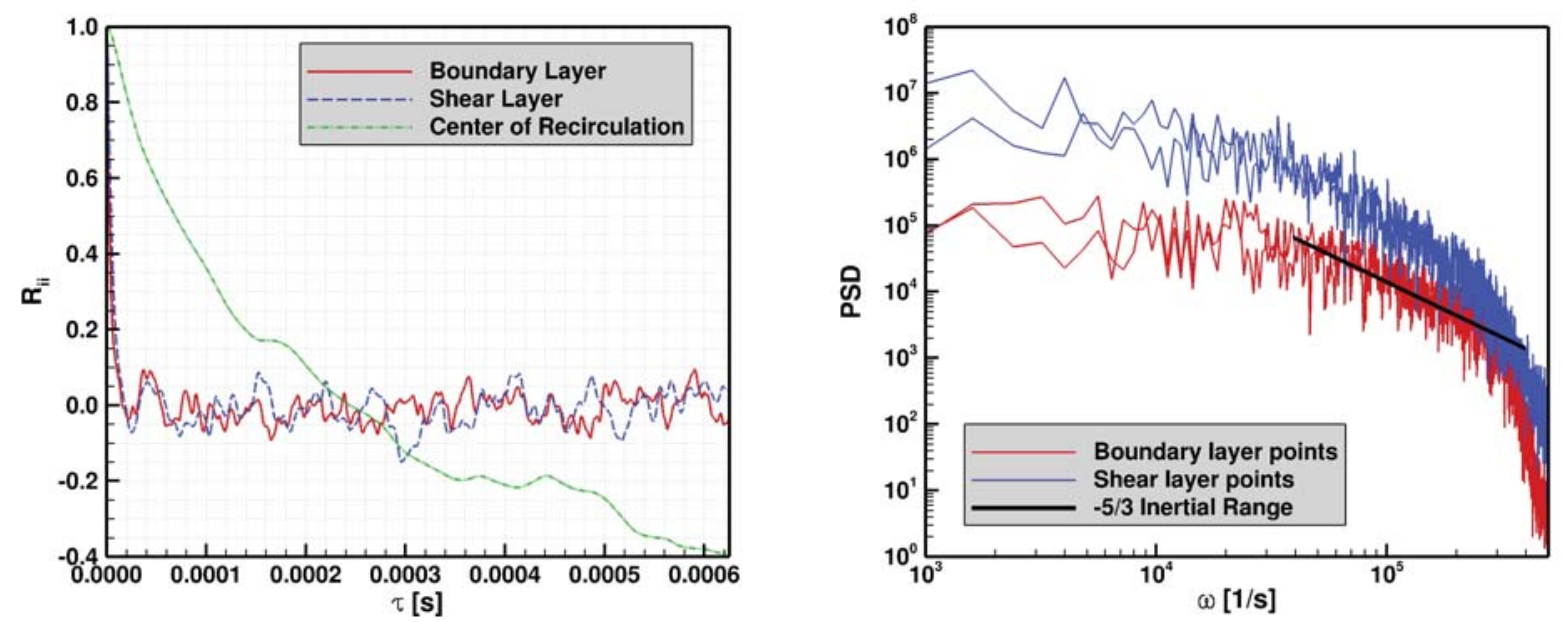

Figure 16: Autocorrelation coefficients at various locations (left), power spectral density at several boundary layer and shear layer locations (right)

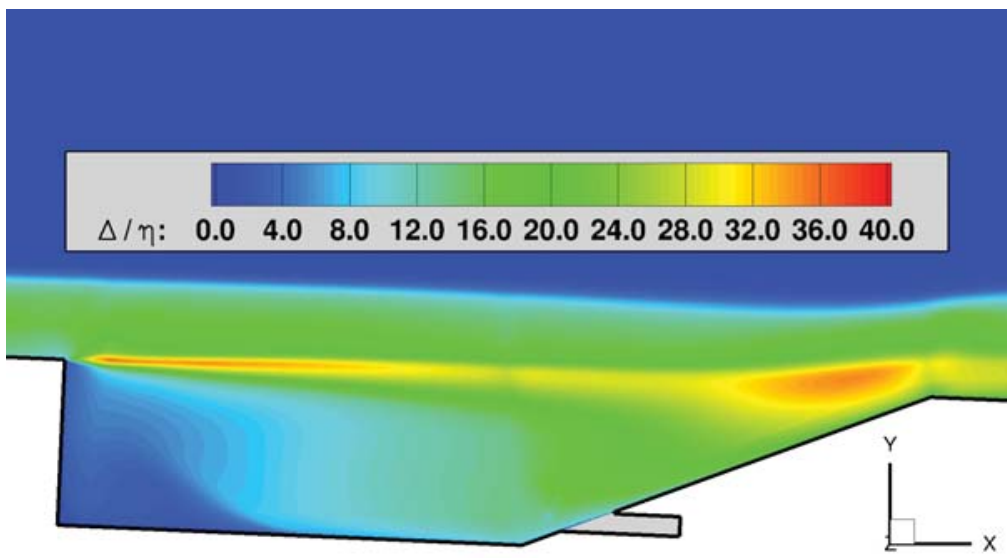

Figure 17: Hybrid RAS/LES filter width divided by the Kolmogorov length scale (based on RAS data)

step) were analyzed, as were two points in the cavity shear layer (before and after the shear layer re-attachment shock wave). The right image of Fig. 16 shows the resulting Power Spectral Density (PSD) for the range of frequencies that were resolved in the coarse grid simulations. An LES should resolve at least a portion of the inertial range of the spectrum, and the slope of this portion of the spectrum is expected to be $-5 / 3$. The PSD image shows a distinct inertial range captured in the spectrum (covering a frequency range of approximately 1 decade) with the expected $-5 / 3$ slope. Hence, even the coarsest grid considered in this effort appears to have the resolution requirements expected for a reasonable LES. Figure 17 displays the ratio of the fine grid filter width to the Kolmogorov dissipation scales (estimated from the Gatski-EAS RAS data). This image provides an estimate of the additional grid refinement that would be required in order to perform a DNS of this flowfield. If one ignores the near-wall boundary layer requirements (which are treated with a RAS framework), then the grid would require up to 100 times more grid cells in each coordinate direction to resolve the smallest turbulence dissipation scales.

As described previously, the sub-grid scale model errors are considered as part of the numerical uncertainty in this effort. If the sub-grid model is performing as intended, then the SGS terms should be modeling the missing physics (that can not be resolved by the grid) in a manner that duplicates the statistics of a fully-resolved DNS. The portion of the SGS terms that deviate from this intended behavior are taken to be lumped with the truncation error of the numerical scheme and evaluated using the GCI metric. This uncertainty is displayed as an uncertainty bar attached to the fine grid results in Fig. 18, which shows the influence of grid resolution on several statistics involving the velocity 
components. The estimated grid-related uncertainty in the streamwise velocity predictions are relatively small, with the largest uncertainty located early in the shear layer development and within the secondary recirculation zone in the cavity. The uncertainty associated with the transverse velocity predictions are considerably larger (relative to the mean value), and are most pronounced within the secondary recirculation zone. This flow feature has even longer time scales than the primary recirculation zone, and the autocorrelation analysis of a point within the primary recirculation zone (see Fig. 16) was found to have an integral time scale 1 to 2 orders of magnitude larger than that of the free shear layer. Hence, it is quite possible that a much longer integration time is required to establish highly accurate statistics in this portion of the flow (much longer than what can be deemed practical).

Both the resolved component and the SGS component of the $2^{\text {nd }}$-order velocity statistics (turbulence kinetic energy and velocity covariance) are displayed in the last two images of Fig. 18. The SGS components are the smaller values (in absolute value) shown in each image. These plots show that the SGS contribution of each $2^{\text {nd }}$-order statistic is considerably smaller than the resolved contribution for each grid level except at the first station shown. At this station the coarse grid SGS components (and to a lesser extent the medium grid values) are comparable to the resolved components. This station is just downstream of the cavity step, and represents a region where the RAS modeled boundary layer transitions to a scale-resolved shear layer. This transition process is accelerated as the grid is refined, as evidenced by the reduction in the SGS components and the escalation of the resolved portion with added grid resolution. As a result, there is a significant variability in the turbulence correlations at the $x=10.43$ inch station as the grid is refined, resulting in the large uncertainty estimates shown. At stations further downstream, the uncertainty is reduced (for the most part) for each of the flow variables. Moreover, at the last two stations the variation between the grid levels is reduced and the differentials become more monotonic in sign as the grid is refined. This observation provides additional confidence in the uncertainty estimates extracted at these stations.

The hybrid RAS/LES velocity statistics extracted from the coarse, medium, and fine grid simulations are compared with the PIV measurements in Fig. 19. The mean streamwise velocity profiles are compared in the top row of images. The simulation results are predicting a considerably thinner shear layer width at the first two axial stations, which is a trend also seen in the pure RAS results. The window size used to post-process the raw PIV data was $0.07 \times 0.07$ inches (10 times coarser in each direction than the resolution of the fine grid simulations). This resolution would not be capable of resolving a shear layer as thin as predicted in the simulations. Hence, it is possible that the difference noted here is simply a matter of implicit filtering introduced by the experimental data processing. With the exception of these first two stations, the agreement between the simulation results and measurements is quite good, although the coarse grid results tend to underpredict the shear layer width. Larger differences are noted in the mean transverse velocity predictions, particularly at the first two axial stations. Recall from Fig. 18 that the uncertainty in the transverse velocity predictions was large in this region of the cavity, and that the autocorrelation analysis suggested that this may be due to an inadequate integration time. The mean transverse velocities are small and have little variability throughout the cavity, so errors from any source will be more noticeable in this parameter. A comparison of transverse velocity predictions with the RAS results from Fig. 9 show an improvement over the Menter model predictions, but no measurable improvement can be claimed over the Gatski-EAS model results. The $2^{\text {nd }}$-order velocity statistics are compared in the last three rows of images. The simulation values shown include both the resolved and modeled SGS components of each correlation. For the most part, the hybrid RAS/LES results are overpredicting the magnitude of the $2^{\text {nd }}$-order velocity statistics. Surprisingly, the Gatski-EAS RAS model predictions almost uniformly outperformed the hybrid RAS/LES predictions for these correlations. However, the hybrid RAS/LES results are encouraging from the standpoint that the spread in the results obtained from each grid level (which includes the uncertainty associated with the performance of the SGS model) is considerably less than the epistemic uncertainty associated with the choice of RAS model. Moreover, the error in the hybrid RAS/LES is reducible (given adequate computational resources). At least for the latter half of the cavity where the uncertainties were deemed quantifiably trustworthy, the uncertainty estimates for the higher order statistics shown in Fig. 18 suggest that finer grids would tend to drive the results in a direction closer to the measured values. 

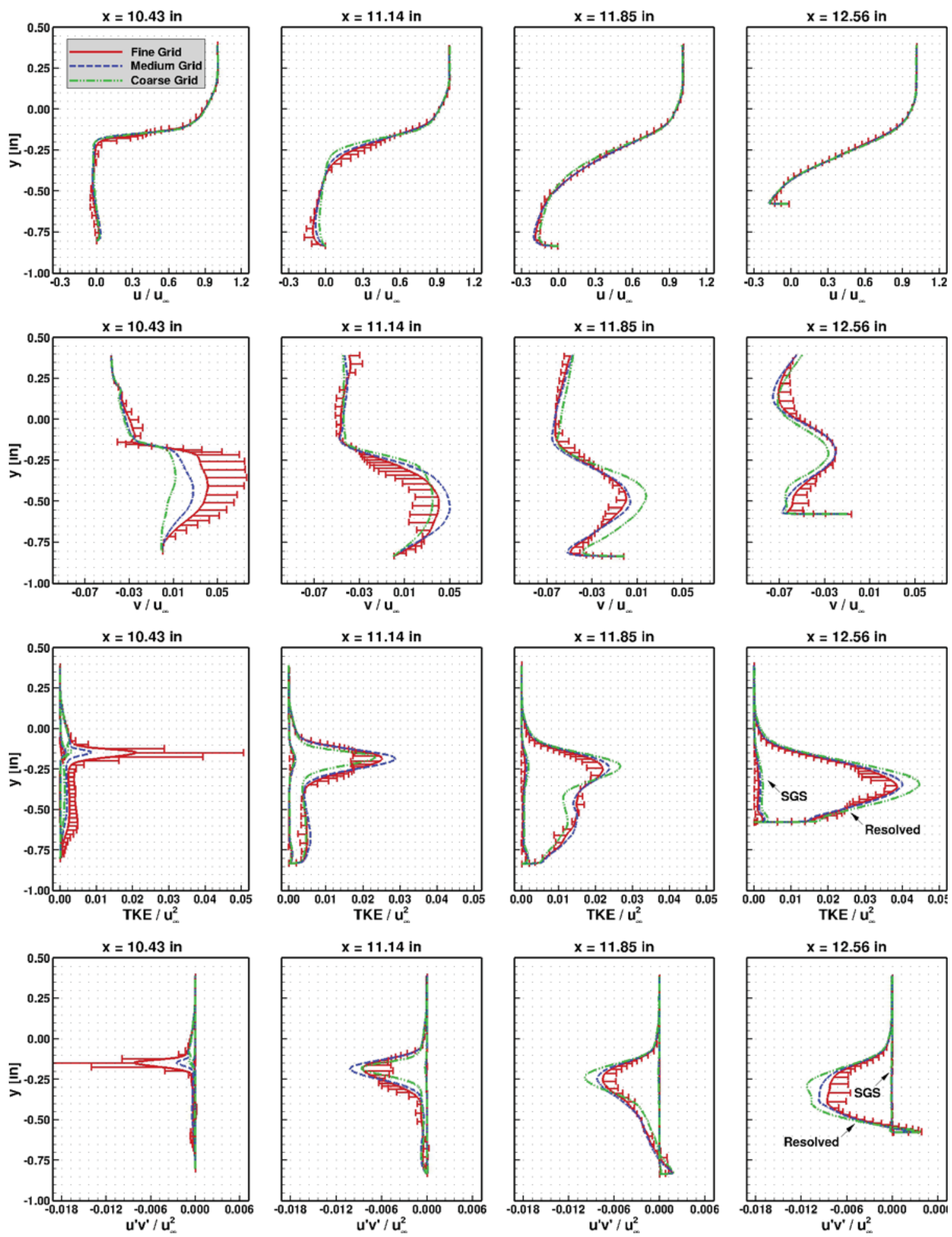

Figure 18: Velocity components, turbulence kinetic energy, and x,y velocity covariance profile comparisons on each grid (error bars show the GCI on the fine grid results) 

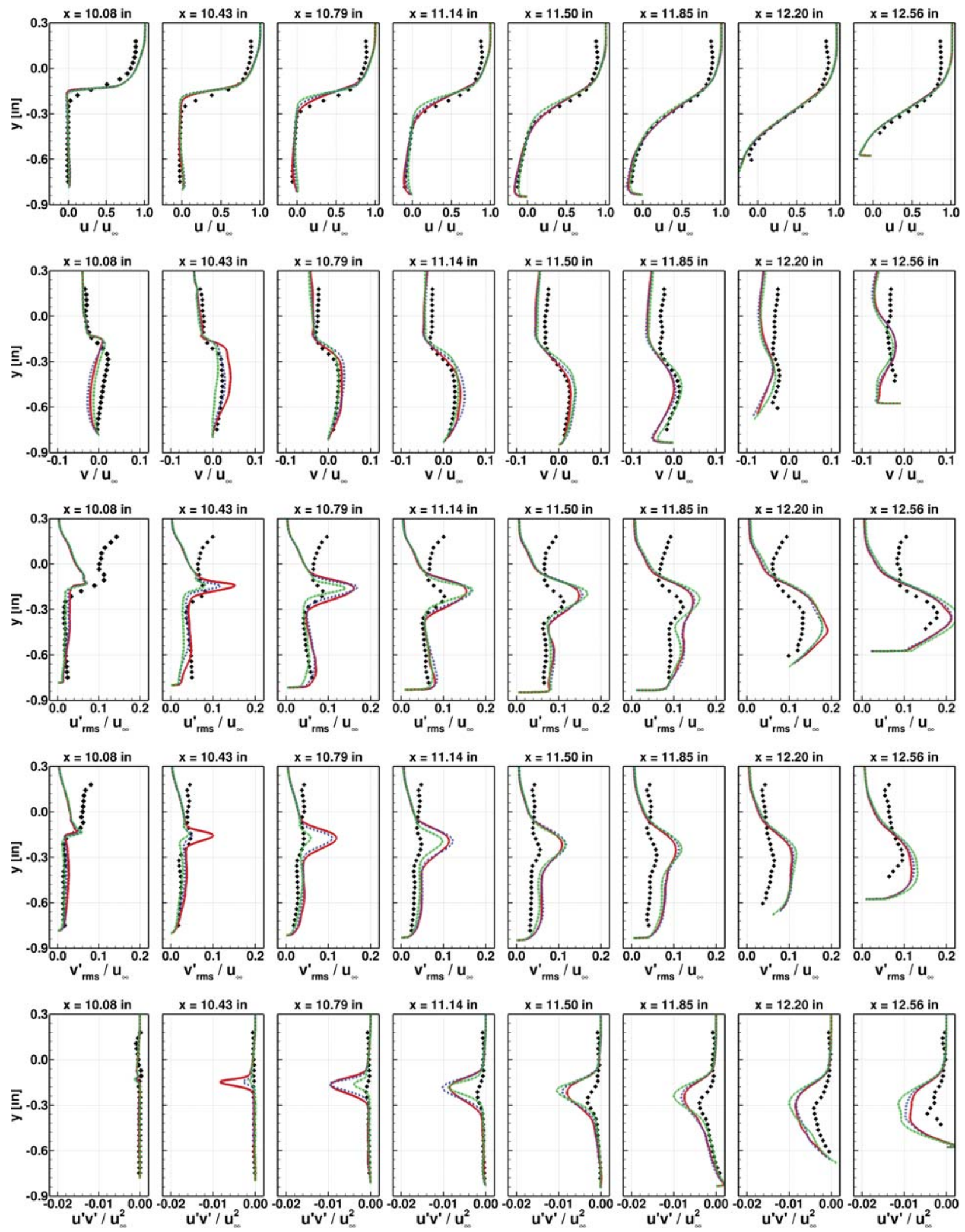

Figure 19: Comparison of hybrid RAS/LES predictions with measurements: PIV measurements (symbols), fine grid (solid red lines), medium grid (dashed blue lines), coarse grid (dash-dot green lines) 


\section{Summary and Future Work}

Reynolds-averaged and hybrid Reynolds-averaged/large-eddy simulations have been performed for a model scramjet cavity flow experiment. Particle Image Velocimetry data was taken at the centerplane of the cavity, allowing for detailed comparisons of the simulated cavity flow structure with measurements. The purpose of the computational effort was to assess the state-of-the-art for both RAS and hybrid RAS/LES predictions for this flowfield, which is a relevant one to the scramjet research community. The hybrid RAS/LES assessment was of particular interest, since recent development activities associated with the VULCAN-CFD code have focused on enhancing these capabilities. As a part of the state-of-the-art assessment, the numerical errors were estimated for each simulation approach.

The Reynolds-averaged simulations of this flow were performed using two linear eddy viscosity models (MenterBSL and Menter-SST $k-\omega$ models) and one non-linear model ( $k-\omega$ based explicit algebraic stress model of Gatski and Rumsey). The numerical errors were shown to be negligibly small relative to the model-form variability associated with the choice of turbulence model. In general, the Menter models did a slightly better job of predicting the mean streamwise velocity profiles within the free shear layer that spans the cavity. However, the explicit algebraic stress model proved to substantially outperform the linear models when predicting all of the other measured properties (mean transverse velocity and velocity variances/covariances). The most prominent feature present in the algebraic stress model that is missing in the linear models is the ability to predict normal Reynolds stress anisotropies. These features have a pronounced effect in applications that involve secondary motions, so it was no surprise that results obtained deviated from the linear eddy viscosity model predictions. However, the drastic improvement noted in the predictions for almost every flow feature analyzed was not expected.

In principle, the hybrid RAS/LES methods have the potential to reduce the uncertainty of RAS (particularly for massively separated flows like the cavity flow considered here) by resolving a substantial fraction of the turbulent flowfield. However, to achieve this improvement one has to ensure that the flowfield is sufficiently grid resolved such that the sub-grid scale model (either explicit or implicit) can reasonably be expected to model the unresolved scales. Assessing whether this goal has been achieved is no easy task due in part to the difficulty with separating pure numerical errors from sub-grid modeling errors. The numerical uncertainty in this effort made no attempt to separate the two, and instead both errors were treated as a common error source that is reducible via grid resolution. The results of this assessment suggest that the overall simulation uncertainty of the scale-resolving predictions (numerical plus sub-grid model) was less than the overall simulation uncertainty (numerical plus turbulence model) of the RAS predictions. In general, the hybrid RAS/LES results showed clear improvements over the RAS predictions that utilized standard linear eddy viscosity models. However, if one assumes that the measurement uncertainty (which was not provided) is small, then the best performing model for this flowfield (in terms of time-averaged statistics) was the explicit algebraic stress RAS model. Future efforts will consider the effects of fuel injection without the effects of combustion. This will introduce an additional modeling issue (the Reynolds mass flux vector) which is known to compromise the RAS closure predictions. The introduction of combustion chemistry will also be considered which complicates the modeling for both the RAS and hybrid RAS/LES efforts.

\section{Acknowledgments}

This effort was funded through the High-Speed Project of the Fundamental Aeronautics Program and carried out at the Hypersonic Airbreathing Propulsion Branch at the NASA Langley Research Center. Computational resources for this work were provided by the NASA Langley Research Center and the NASA Advanced Supercomputing (NAS) Division. The author would like to thank Dr. Tomasz Drozda for many helpful discussions pertaining to this research effort. The author would also like to acknowledge the efforts of Dr. Steven Tuttle from the Naval Research Laboratory for graciously providing the experimental data for this study, and Dr. David Peterson from the Air Force Research Laboratory for providing the geometry and other information based on his simulations of this flowpath.

\section{References}

[1] Slotnick, J. Khodadoust, A., Alonso, J., Darmofal, D., Gropp, W., Lurie, E., and Mavriplis, D., "CFD Vision 2030 Study: A Path to Revolutionary Computational Aerosciences," NASA Contractor Report 218178, 2014.

[2] Baurle, R. A., Tam, C.-J., Edwards, J. R., and Hassan, H. A., "Hybrid Simulation Approach for Cavity Flows: Blending, Algorithm, and Boundary Treatment Issues," AIAA Journal, Vol. 41, No. 8, 2003, pp. 1463-1480.

[3] Menter, F. R. and Kuntz, M., "Adaptation of Eddy-Viscosity Turbulence Models to Unsteady Separated Flow Behind Vehicles," The Aerodynamics of Heavy Vehicles: Trucks, Buses, and Trains, edited by B. F. McCallen, R. and J. Ross, Springer Berlin Heidelberg, 2004, pp. $339-352$.

[4] Spalart, P. R., Deck, S., Shur, M. L., Squires, K. D., Strelets, M. K., Travin, A. K., "A New Version of Detached-Eddy Simulation, Resistant to 
Ambiguous Grid Densities," Theoretical Computational Fluid Dynamics, Vol. 20, No. 3, 2006, pp. 181-195.

[5] Girimaji, S. S., "Partially-Averaged Navier-Stokes Model for Turbulence: A Reynolds-Averaged Navier-Stokes to Direct Numerical Simulation Bridging Method," Journal of Applied Mechanics, Vol. 73, No. 3, 2006, pp. 413-421.

[6] White, J. A. and Morisson, J. H., "Pseudo-Temporal Multi-Grid Relaxation Scheme for Solving the Parabolized Navier-Stokes Equations," AIAA Paper 99-3360, 1999.

[7] VULCAN, "http://vulcan-cfd.larc.nasa.gov/," 2014.

[8] White, J. A, Baurle, R. A., Fisher, T. C., Quinlan, J. R., and Black, W. S., "Low Dissipation Advection Schemes Designed for Large Eddy Simulations of Hypersonic Propulsion Systems," AIAA Paper 2012-4263, 2012.

[9] Quinlan, J., McDaniel, J., and Baurle, R., "Simulation of a Wall-Bounded Flow Using a Hybrid LES/RAS Approach with Turbulence Recycling," AIAA Paper 2012-3285, 2012.

[10] Tuttle, S., Carter, C., and Hsu, K., "Particle Image Velocimetry in an Isothermal and Exothermic High-Speed Cavity," AIAA Paper 2012-0330, 2012.

[11] Grady, N., Frankland, J. Pitz, R., Carter, C., and Hsu, K., "UV Raman Scattering Measurements of Supersonic Reacting Flow Over a Piloted, Ramped Cavity," AIAA Paper 2012-0614, 2012.

[12] Peterson, D. M., Hagenmaier, M. A., Carter, C. D., and Tuttle, S. G., "Hybrid Reynolds-Averaged and Large-Eddy Simulations of a Supersonic Cavity Flameholder," AIAA Paper 2013-2483, 2013.

[13] Peterson, D. M., Hassan, E. A., Tuttle, S. G., Hagenmaier, M. A., and Carter, C. D., "Numerical Investigation of a Supersonic Cavity Flameholder," AIAA Paper 2014-1158, 2014.

[14] Roache, P. J., Verification and Validation in Computational Science and Engineering, Hermosa Publishers, 1998.

[15] Litton, D. K., Edwards, J. R., and White, J. A., "Algorithm Enhancements to the VULCAN Navier-Stokes Solver," AIAA Paper 2003-3979, 2003.

[16] Edwards, J. R., "A Low Diffusion Flux-Splitting Scheme for Navier-Stokes Calculations,” Computers E Fluids, Vol. 26, No. 6, 1997 , pp. 635-659.

[17] van Leer, B., "Towards the Ultimate Conservation Difference Scheme. II. Monotinicity and Conservation Combined in a Second Order Scheme," Journal of Computational Physics, Vol. 14, 1974, pp. 361-370.

[18] McBride, B. J. and Gordon, S., "Computer Program for Calculation of Complex Chemical Equilibrium Composition and Applications, I. Analysis," NASA Reference Publication 1311, 1994.

[19] McBride, B. J. and Gordon, S., "Computer Program for Calculation of Complex Chemical Equilibrium Composition and Applications, II. Users Manual and Program Description," NASA Reference Publication 1311, 1996.

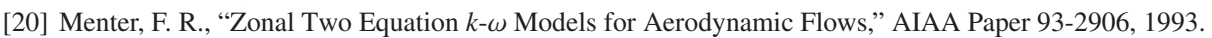

[21] Rumsey, C. L. and Gatski, T. B., "Summary of EASM Turbulence Models in CFL3D with Validation Test Cases," NASA Technical Report TM2003-212431, 2003.

[22] Huang, P. G., "Physics and Computations of Flows with Adverse Pressure Gradients," Modeling Complex Turbulent Flows, edited by M. D. Salas, J. N. Hefner, and L. Sakell, Vol. 7 of ICASE/LaRC Interdisciplinary Series in Science and Engineering, Springer Netherlands, 1999 , pp. $245-258$.

[23] Choi, J.-L., Edwards, J. R., and Baurle, R. A., "Compressible Boundary Layer Predictions at High Reynolds Number Using Hybrid LES/RANS Methods," AIAA Journal, Vol. 47, No. 9, 2009, pp. 2179-2193.

[24] Boles, J. A., Edwards, J. R., Choi, J.-L., and Baurle, R. A., "Simulations of High-Speed Internal Flows Using LES/RANS Models," AIAA Paper 2009-1324, 2009.

[25] Yoshizawa, A. and Horiuti, K., "A Statistically-Derived Subgrid Scale Kinetic Energy Model for Large-Eddy Simulation of Turbulent Flows," Journal of the Physical Society of Japan, Vol. 54, 1985, pp. 2834-2839.

[26] Spalart, P. R., Jou, W.-H., Strelets, M. K., and Allmaras, S. R., "Comments on the Feasibility of LES for Wings, and on a Hybrid RANS/LES Approach," 1st AFOSR International Conference on DNS/LES (invited), 1997.

[27] Strelets, M. K., "Detached Eddy Simulation of Massively Separated Flows," AIAA Paper 2001-0879, 2001.

[28] Shur, M. L., Spalart, P. R., Strelets, M. K., and Travin, A. K., "A Hybrid RANS-LES Approach with Delayed-DES and Wall-Modelled LES Capabilities," International Journal of Heat and Fluid Flow, Vol. 29, No. 6, 2008, pp. 1638-1649.

[29] Nikitin, N. V., Nicoud, F., Wasistho, B. Squires, K. D., and Spalart, P. R., "An Approach to Wall Modeling in Large-Eddy Simulations," Physics of Fluids, Vol. 12, 2000, pp. 1629-1632.

[30] Colella, P. and Woodward, P., "The Piecewise Parabolic Method (PPM) for Gasdynamical Simulations," Journal of Computational Physics, Vol. 54, No. 1, 1984, pp. 174-201.

[31] Yamaleev, N. K. and Carpenter M. H., "High-order Energy Stable WENO Schemes," AIAA Paper 2009-1135, 2009.

[32] Fisher, T. C., Carpenter, M. H., Yamaleev, N. K., and Frankel, S. H., "Boundary Closures for Fourth-order Energy Stable Weighted Essentially Non-oscillatory Finite Difference Schemes," Journal of Computational Physics, Vol. 230, No. 1, 2011, pp. 3727-3752.

[33] Ducros, F. Ferrand, V., Nicoud, F., Weber, C., Darracq, D., Gacherieu, C., and Poinsot, T., "Large-Eddy Simulation of the Shock/Turbulence Interaction," Journal of Computational Physics, Vol. 152, No. 2, 1999, pp. 517-549.

[34] Larsson, J., "Large Eddy Simulations of the HyShot II Scramjet Combustor Using a Supersonic Flamelet Model," AIAA Paper 2012-4261, 2012.

[35] Suresh, A. and Huynh, H. T., "Numerical Experiments on a New Class of Nonoscillatory Schemes," AIAA Paper 92-0421, 1992.

[36] Krist, S. L., Biedron, R. T., and Rumsey, C. L., "CFL3D User's Manual (Version 5.0)," NASA Technical Report TM-1998-208444, 1998.

[37] Urbin, G., Knight, D., and Zheltovodov, A. A., "Large Eddy Simulation of a Supersonic Boundary Layer Using Unstructured Grids," AIAA Journal, Vol. 39, No. 7, 2001, pp. 1288-1295.

[38] Tennekes, H. and Lumley, J. L., A First Course in Turbulence, MIT Press, 1972. 